\title{
Bargmann transform, Zak transform, and coherent states
}

\author{
A. J.E. M. Janssen \\ California Institute of Technology, Pasadena, California 91125 \\ (Received 7 November 1980; accepted for publication 11 March 1981)
}

\begin{abstract}
It is well known that completeness properties of sets of coherent states associated with lattices in the phase plane can be proved by using the Bargmann representation or by using the $k q$ representation which was introduced by $\mathbf{J}$. Zak. In this paper both methods are considered, in particular, in connection with expansions of generalized functions in what are called Gabor series. The setting consists of two spaces of generalized functions (tempered distributions and elements of the class $S^{*}$ ) which appear in a natural way in the context of the Bargmann transform. Also, a thorough mathematical investigation of the Zak transform is given. This paper contains many comments and complements on existing literature; in particular, connections with the theory of interpolation of entire functions over the Gaussian integers are given.
\end{abstract}

PACS numbers: 02.30.Mv, 02.30.Lt

\section{INTRODUCTION}

If $x \in \mathbb{R}, y \in \mathbb{R}$, then $G(x, y)$ denotes the function $(G(x, y))(t)=2 \exp \left(-\pi(t-x)^{2}+2 \pi i y t-\pi i x y\right) \quad(t \in \mathbb{R}) ;$ $G(x, y)$ is called a coherent state, ${ }^{1,2}$ or also a Gabor function. ${ }^{3,4}$ In the past ten years a number of papers ${ }^{1,2,4-7}$ appeared about the completeness of the collection $\{G(n \alpha, m \beta) \mid n, m$ integers $\}$ where $\alpha>0, \beta>0$. These papers deal with the following question: if $f$ is a (generalized) function and $(f, G(n \alpha, m \beta))=0$ for all integers $n$ and $m$, then does it follow that $f \equiv 0$ ? As early as 1932 , von Neumann ${ }^{8}$ noticed (apparently without publishing a proof) that the answer is "yes" if $f \in L^{2}(\mathbb{R}), \alpha \beta=1$. Two proofs of this fact were given in 1970 by using the Bargmann transform, ${ }^{9}$ and in 1975 a proof was given by using the $k q$ representation. The most complete answer to the above question was probably given in 1979. It is shown ${ }^{7}$ that $(f, G(n \alpha, m \beta))=0$ for all integers $n$ and $m$ implies $f \equiv 0$ for a very large class of generalized functions $f$ whenever $\alpha \beta<1$. Also, in case $\alpha \beta=1$, a characterization of all tempered distributions $f$ with $(f, G(n \alpha, m \beta))$ $=0$ for all $n$ and $m$ is given. The main tools are a PhragménLindelöf theorem and the Bargmann transform, although the latter is not explicitly mentioned.

A related question concerns expansion of (generalized) functions $f$ in series of the form $\Sigma_{n, m} c_{n m} G(n \alpha, m \beta)$ with $\alpha \beta=1$ (Gabor series). In 1946 Gabor $^{10}$ suggested a simultaneous time-frequency analysis of signals based on these expansions. In 1979 existence and uniqueness theorems about Gabor series were given (cf. Ref. 6, where expressions for the coefficients $c_{n m}$ are given, and Ref. 4 , where existence of Gabor's expansions for tempered distributions is proved; in both papers the $k q$ representation, although not explicitly mentioned, plays an important role).

We give a survey of the content of this paper. In Sec. 2 we consider the spaces $S$ of smooth functions and $S^{*}$ of generalized functions, and we show that, in connection with the Bargmann transform, these spaces arise in a natural way. In Sec. 3 the Zak transform $T$, which maps functions $f$ of one real variable onto functions $T f$ defined on the unit square, is introduced and studied in detail. A peculiar property of the Zak transform ${ }^{11}$ is the following one: if $f \in L^{2}(\mathbb{R})$ and $T f$ is continuous, then $T f$ has a zero in the unit square. In Sec. 4 a number of consequences of this property are given. One of the consequences is that one can improve the convergence of Gabor series (which, in general, converge not even in $L^{2}$ sense for elements of $S$ ) by shifting the lattice over a distance $(a, b)$ with suitably chosen numbers $a$ and $b$. Also, the results about completeness after deleting one or more coherent states $^{2,5.7}$ are completed and generalized, and a relation with classical results in interpolation theory is indicated. Although almost all results deal with square lattices of unit area with axes parallel to the $x$ and $y$ axis in the phase plane, some indications are given how to handle general lattices. Finally, the paper shows existence of Gabor's expansion for elements of $S^{*}$.

\section{THE SPACES $S$ AND $S{ }^{*}$ AND THE BARGMANN TRANSFORM}

In 1961 Bargmann ${ }^{12}$ constructed a unitary mapping of $L^{2}(\mathbb{R})$ onto the set $\mathrm{F}$ of all entire functions $f$ of growth $\leqslant\left(2, \frac{1}{2}\right)$ for which $\int_{\mathbb{C}}|f(z)|^{2} e^{-|z|^{2}} d z<\infty$. On the space $F$ Fock's solution $\xi=\partial / \partial \eta$ of the commutation rule $[\xi, \eta]=1$ is realized. In 1967 Bargmann ${ }^{13}$ described several spaces of test functions and generalized functions in terms of certain subsets of $F$ and duals of these. In particular the spaces $S$ and $S^{\prime}$ (Schwartz's space of functions of rapid decrease and of tempered distributions respectively) were considered. In this section we shall investigate the relation between Bargmann transform and the spaces $S$ and $S^{*}$ (of smooth and generalized functions respectively) which were introduced in Ref. 14 and studied extensively in Refs. 3, 15, and 16.

2.1. The space $S$ consists of all entire functions $f$ for which there are $M>0, A>0, B>0$ such that

$$
\left(^{*}\right)|f(x+i y)| \leqslant M \exp \left(-\pi A x^{2}+\pi B y^{2}\right) \quad(x \in \mathbb{R}, y \in \mathbb{R}) .
$$

A sequence $\left(f_{n}\right)_{n}$ in $S$ converges to zero in $S$ sense $\left(f_{n} \stackrel{s}{\rightarrow}\right)$ if there are $M>0, A>0, B>0$ such that $\left({ }^{*}\right)$ holds for all $f_{n}$ and such that $f_{n} \rightarrow 0$ pointwise. The space $S *$ consists of all continuous antilinear functionals defined on $S$. The action of $F \in S^{*}$ on $f \in S$ is denoted by $(F, f)$. A sequence $\left(F_{n}\right)_{n}$ in $S^{*}$ 
converges to zero in $S^{*}$ sense $\left(F_{n} \stackrel{s^{*}}{\rightarrow} 0\right)$ if $\left(F_{n}, f\right) \rightarrow 0$ for all $f \in S$. Note that $G(x, y) \in S$ for all $x \in \mathbb{R}, y \in \mathbb{R}$.

2.2. For $n=0,1, \cdots, \psi_{n}$ denotes the $n$th Hermite function. We choose our normalizations such that ${ }^{17}$

$$
\exp \left(\pi x^{2}-2 \pi(x-w)^{2}\right)=\sum_{n=0}^{\infty} c_{n} w^{n} \psi_{n}(x) \quad(x \in \mathbb{C}, w \in \mathbb{C})
$$

where $c_{n}=2^{-1 / 4}(4 \pi)^{n / 2} /(n !)^{1 / 2}$ for all $n$. We have $\psi_{n} \in S$.

There is a one-to-one correspondence between the space $S$ and the space $D$ of all complex sequences $\left(a_{n}\right)_{n}$ with $a_{n}=0\left(e^{-n \epsilon}\right)$ for some $\epsilon>0$; if $f \in S$, then $\left(\left(f, \psi_{n}\right)\right)_{n} \in D$, and if $\left(a_{n}\right)_{n} \in D$, then $\Sigma_{n} a_{n} \psi_{n}$ converges in $S$ sense to an element of $S$. There is a similar correspondence between the space $S^{*}$ and the space $D^{*}$ of all complex sequences $\left(b_{n}\right)_{n}$ with $b_{n}=0\left(e^{n \epsilon}\right)$ for all $\epsilon>0$. It follows ${ }^{18}$ that $S \subset S$ and that $S^{\prime} \subset S^{*}$.

2.3. A different way to describe the space $S$ is the following one: In Ref. 14 the space $S_{\beta}^{\alpha}(\alpha>0, \beta>0)$ is defined as the set of all functions $f: \mathbb{R} \rightarrow \mathbb{C}$ for which there exist $C>0, A>0$, $B>0$ such that

$$
\left({ }^{* *}\right)\left|x^{k} f^{(q)}(x)\right| \leqslant C A^{k} B^{q} k^{k \alpha} q^{q \beta},
$$

for all $x \in \mathbb{R}, k=0,1, \cdots, q=0,1, \cdots$. Our space $S$ can be identified with $S_{1 / 2}^{1 / 2}$ as follows: If $f \in S$, then the restriction of $f$ to $\mathbb{R}$ satisfies inequalities as in $\left({ }^{*}\right)$, and if we have an $f: \mathbb{R} \rightarrow \mathbb{C}$ satisfying inequalities as in $(* *)$, then $f$ can be extended to an entire function satisfying an inequality as in $2.1\left(^{*}\right)$. Also, the notions of convergence in 2.1 for $S$ and in Ref. 14 for $S_{1 / 2}^{1 / 2}$ can be shown to be equivalent.

We note some topological properties of the spaces $S$ and $S^{*}$. If we consider $S$ and $S^{*}$ with the weak * topologies, i.e., with the linear topologies generated by all sets of the form $\{f \in S \mid(F, f) \in 0\}$ (where $F \in S^{*}, 0 \subset \mathbb{C}$ open) and $\left\{F \in S^{*} \mid\right.$ $(F, f) \in 0\}$ (where $f \in S, 0 \subset \mathbb{C}$ open), then the dual of $S$ is $S^{*}$ and the dual of $S^{*}$ is $S$. The space $S\left(S^{*}\right)$ is complete in the sense that if $f_{n} \in S\left(F_{n} \in S^{*}\right)$ and $\lim _{n \rightarrow \infty}\left(F, f_{n}\right)\left[\lim _{n \rightarrow \infty}\left(F_{n}, f\right)\right]$ exists for all $F \in S^{*}(f \in S)$, then there is an $f \in S\left(F \in S^{*}\right)$ such that $(F, f)=\lim _{n \rightarrow \infty}\left(F, f_{n}\right)\left[(F, f)=\lim _{n \rightarrow \infty}\left(F_{n}, f\right)\right]$ for all $F \in S^{*}$ $(f \in S)$. More information can be found in Ref. 15 .

To indicate how big the space $S^{*}$ is, we observe that any measurable $F: \mathbb{R} \rightarrow \mathbb{C}$ for which $\int_{-\infty}^{\infty} \exp \left(-\epsilon t^{2}\right)|F(t)| d t$ $<\infty$ for all $\epsilon>0$ can be regarded as an element of $S^{*}$ by putting $(F, f):=\int_{-\infty}^{\infty} F(t) \overline{f(t)} d t$ for $f \in S$.

2.4. We give a list of operators of $S$ : if $f \in S, a \in \mathbb{C}, b \in \mathbb{C}$, $\alpha>0$, then

$$
\begin{aligned}
\left(T_{a} f\right)(t) & =f(t+a), \quad\left(R_{b} f\right)(t)=e^{-2 \pi i b t} f(t), \\
\left(N_{\alpha} f\right)(t) & =\left(\frac{1}{\sinh \alpha}\right)^{1 / 2} \\
\times \int_{-\infty}^{\infty} \exp \left(\frac{-\pi}{\sinh \alpha}\left(\left(t^{2}+z^{2}\right) \cosh \alpha-2 t z\right)\right) f(z) d z, & \\
(\mathscr{F} f)(t) & =\int_{-\infty}^{\infty} e^{-2 \pi i t z} f(z) d z,\left(\mathscr{F}^{*} f\right)(t)=(\mathscr{F} f)(-t), \\
(P f)(t) & =(1 / 2 \pi i) f^{\prime}(t), \quad(Q f)(t)=t f(t),
\end{aligned}
$$

for $t \in \mathbb{C} .{ }^{19}$ These operators are continuous and have adjoints that map $S$ into $S$; they can therefore be extended to continu- ous linear operators of $S^{*} .^{20}$

2.5. Definition: For $F \in S^{*}$ the Bargmann transform $B F$ of $F$ is defined by

$$
(B F)(z)=e^{i \pi z^{2}}\left(T_{z} F, g\right) \quad(z \in \mathbb{C}) .
$$

Here $g(t)=2^{1 / 4} \exp \left(-\pi t^{2}\right)$ for $t \in \mathbb{C}$.

2.6. The following formula (for $F \in \mathrm{S}^{\prime}$ ) is due to Bargmann ${ }^{12}$; for the sake of completeness we give a proof.

Theorem: Let $F \in S^{*}$. Then

$$
(B F)(z)=\sum_{n=0}^{\infty} \frac{\left(F, \psi_{n}\right)}{(n !)^{1 / 2}}\left(\pi^{1 / 2} z\right)^{n} \quad(z \in \mathbb{C}) .
$$

Proof: Put $h_{z}(t)=2^{1 / 4} \exp \left(\frac{1}{2} \pi z^{2}-\pi(t-z)^{2}\right)$ for $z \in \mathbb{C}$ $t \in \mathbb{C}$. Since $(B F)(z)=\left(f, h_{\bar{z}}\right)$ and

$h_{2}(t)=2^{1 / 4} \exp \left(\pi t^{2}-2 \pi\left(t-\frac{1}{2} z\right)^{2}\right)=\sum_{n=0}^{\infty} \frac{\psi_{n}(t)}{(n !)^{1 / 2}}\left(\pi^{1 / 2} z\right)^{n}$,

with convergence in $S$ sense for every $z \in \mathbb{C}$, we have

$$
(B F)(z)=\left(F, h_{\bar{z}}\right)=\sum_{n=0}^{\infty} \frac{\left(F, \psi_{n}\right)}{(n !)^{1 / 2}}\left(\pi^{1 / 2} z\right)^{n}
$$

2.7. Let $\mathscr{E}$ be the space of all entire functions of growth $\leqslant(2, \pi / 2)$, and let $\mu$ be the measure on $\mathbb{C}$ defined by $d \mu(z)=e^{-\pi|z|^{2}} d z$. If $\mathscr{H}=\mathscr{C} \cap L^{2}(\mathbb{C}, \mu)$, then $\mathscr{H}$ is a Hilbert space for which $\left(\pi^{i n} z^{n} / V n !\right)_{n}$ is a complete orthonormal system, and $B$ maps $L^{2}(\mathbb{R})$ isometrically onto $\mathscr{H} .^{12}$ Also, ${ }^{13}$

$$
\begin{aligned}
B(\mathrm{~S}) & =\left\{f \in \mathscr{C} \mid f(z) \exp \left(-\frac{1}{2} \pi|z|^{2}\right)\right. \\
& \left.=O\left((1+|z|)^{-N}\right) \text { for all } N>0\right\}, \\
B(S) & =\left\{f \in \mathscr{C} \mid f(z) \exp \left(-\frac{1}{2} \pi|z|^{2}\right)\right. \\
& \left.=O\left((1+|z|)^{N}\right) \text { for some } N>0\right\} .
\end{aligned}
$$

Theorem: (i) $B(S)=\{f \in \mathscr{E} \mid$ growth of $f<(2, \pi / 2\}$, (ii) $B\left(S^{*}\right)=\mathscr{E}$.

Proof: Let $F \in S$. There is an $\epsilon>0$ such that $\left(F, \psi_{n}\right)=O\left(e^{-n \epsilon}\right)$. Hence, by Stirling's formula

$$
\left[\left(F, \psi_{n}\right) /(n !)^{1 / 2}\right] \pi^{n / 2}=O\left(n^{-1 / 4}(\pi / n)^{n / 2} e^{-n(\epsilon+1)}\right) .
$$

It follows from Ref. 21 , Theorem 2.2.2 that $B f$ has growth $<(2, \pi / 2)$. Conversely, let $f \in \mathscr{C}$, growth of $f<(2, \pi / 2)$. Writing $f(z)=\Sigma_{n} a_{n} z^{n}$ we know from Ref. 21, 2.2.10 that lim sup $n\left|a_{n}\right|^{2 / n}<\pi e$. Hence $b_{n}:=a_{n} \pi^{n / 2}(n !)^{1 / 2}=O\left(e^{-n \epsilon}\right)$ for some $\epsilon>0$. So if we put $F=\Sigma_{n} b_{n} \psi_{n}$, then $F \in S$ and $B F=f$.

The proof of (ii) is similar.

Remarks: (1) There are similar characterizations for the elements of $B(\mathscr{C})$ and $B(\mathscr{M})(\mathscr{C}$ is the convolution class and $(\mathscr{M})$ is the multiplication class; cf. Ref. 16). It may be shown that

$$
\begin{aligned}
B(\mathscr{C}) & =\left\{f \in \mathscr{C} \mid \forall_{p>1} \exists_{q<1}[f(x+i y)\right. \\
& \left.\left.=O\left(\exp \left(\pi q x^{2}+\pi p y^{2}\right)\right)\right]\right\}, \\
B(\mathrm{M}) & =\{f \in \mathscr{C}\} \mid \forall_{p>\frac{1}{2}} \exists_{q<!}[f(x+i y) \\
& \left.\left.=O\left(\exp \left(\pi q y^{2}+\pi p x^{2}\right)\right)\right]\right\} .
\end{aligned}
$$

(2) Theorem 2.7 shows that Theorem 2.8 in Ref. 7 is in some sense the best possible result that can be obtained by using the Bargmann transform.

2.8. In the list below we have $F \in S^{*}, a \in \mathbb{C}, b \in \mathbb{C}, \alpha>0$, $z \in \mathbb{C}$.

(1) $\left(B T_{a} F\right)(z)=e^{-! \pi a^{2}-\pi a z}(B F)(z+a)$,

(2) $\left(B R_{b} F\right)(z)=e^{-1 \pi b^{2}-\pi i b z}(B F)(z-i b)$, 


$$
\begin{aligned}
& \text { (3) }\left(B N_{\alpha} F\right)(z)=e^{-\lfloor\alpha}(B F)\left(e^{-\alpha} z\right), \\
& \text { (4) }(B \mathscr{F} F)(z)=(B F)(i z), \\
& \text { (5) }(B P F)(z)=\frac{1}{2} i z(B F)(z)+(1 / 2 \pi i)(B F)^{\prime}(z), \\
& \text { (6) }(B Q F)(z)=\frac{1}{2} z(B F)(z)+(1 / 2 \pi)(B F)^{\prime}(z), \\
& \text { (7) }(B(Q-i P) F)(z)=z(B F)(z), \\
& \text { (8) }(B(Q+i P) F)(z)=(1 / \pi)(B F)^{\prime}(z), \\
& \text { (9) }\left(B\left(Q^{2}+P^{2}\right) F\right)(z)=(1 / 2 \pi)(B F)(z)+(z / \pi)(B F)^{\prime}(z) .
\end{aligned}
$$

The proofs of these formulas are straightforward; compare also Ref. 12 where $B U$ is calculated with $U$ a canonical operator associated with a symplectic transformation of the phase plane, and Ref. 3, Sec. 27.3.

The obvious advantage of the space $S^{*}$ over $\mathscr{S}^{\prime}$ is that we can consider in (1) and (2) complex values of $a$ and $b$. The obvious disadvantage is the fact that $S^{*}$ is described in terms of entire functions so that its elements are hard to localize. Nevertheless, it appears that one can say at least something ${ }^{22}$ about the carriers of the elements of $S^{*}$ with the aid of the Bargmann transform and the theory of analytic functionals.

Some other useful formulas are

$$
\begin{aligned}
& (B G(x, y))(z)=\exp \left(-\frac{1}{2} \pi\left(x^{2}+y^{2}\right)+\pi(x+i y) z\right) \quad(z \in \mathbb{C}), \\
& \left(B \delta_{0}^{(k)}\right)(z)=(-1)^{k}(k !)^{1 / 2}(2 \pi)^{k / 2} \psi_{k}(z / \sqrt{ } 2) \quad(z \in \mathbb{C})
\end{aligned}
$$

for $k=0,1, \cdots$. For $F \in S^{*}, f \in S, a \in \mathbb{R}, b \in \mathbb{R}$,

$$
\begin{aligned}
& (F, G(a, b))=\exp \left(-\frac{1}{2} \pi\left(a^{2}+b^{2}\right)\right)(B F)(a-i b), \\
& (F, f)=\int_{\mathbb{C}} e^{-\pi|z|^{2}}(B F)(z) \overline{(B f)(z)} d z,
\end{aligned}
$$

so that (integration over $\mathbf{R}^{2}$ )

$$
(F, f)=\iint(F, G(a, b))(G(a, b), f) d a d b,
$$

which agrees with the formula 27.12.1.5 in Ref. 3 .

\section{THE ZAK TRANSFORM}

In this section we study the Zak transform which was introduced in 1967 by Zak to construct a quantum mechanical representation ( $k q$ representation) for the description of the motion of a Bloch electron in the presence of a magnetic or electric field. ${ }^{23-25}$ This representation can also be used for the quantum mechanical description of angle and phase. ${ }^{26}$ The Zak transform $T$ maps functions $f$ defined on $\mathbb{R}$ onto functions $T f$ of two variables as follows:

$$
(T f)(z, w)=\sum_{n=-\infty}^{\infty} f(z-n) e^{-2 \pi i n w}
$$

Zak denotes the first variable (quasiposition variable) by $q$ and the second variable (quasimomentum variable) by $k$. We consider here $T$ as a mapping of $L^{2}(\mathbb{R})$ into $L^{2}\left([0,1]^{2}\right)$, and also as a mapping of $S^{*}$ into $S^{2^{*}}$ [and of $S^{\prime}$ into $\left(S^{2}\right)^{\prime}$ ]. Although the Zak transform looks, at first sight, less interesting from the mathematical point of view than does the Bargmann transform, it pays (as we shall see in the next section) to investigate its properties systematically. A striking property is that $T f$ has a zero in $[0,1]^{2}$, provided that $T f$ is continuous. We further give a formula for the product $T F \cdot \overrightarrow{T f}$ (in case this makes sense) which is very convenient when prov- ing completeness properties, and we determine $T(S), T\left(S^{*}\right)$, $T(\mathrm{~S})$, and $T\left(\mathrm{~S}^{\prime}\right)$.

$$
\text { 3.1. Definition: Let } F \in S^{*} \text {. We define }
$$

$$
T F:=\sum_{n=-\infty}^{\infty} T_{-n}^{(1)} R_{n}^{(2)}(F \otimes H)
$$

where $H \equiv 1$ [for the definition of the tensor product, cf. Ref. 15, Appendix 1, 1.17; we have $\left(F_{1} \otimes F_{2}, f_{1} \otimes f_{2}\right)$

$=\left(F_{1}, f_{1}\right)\left(F_{2}, f_{2}\right)$ for $\left.F_{i} \in S^{*}, f_{i} \in S(i=1,2)\right]$. This definition makes sense, for if $F \in S^{*}, f_{1} \in S, f_{2} \in S$, then

$$
\begin{aligned}
& \sum_{n=-\infty}^{\infty}\left(T_{-n}^{(1)} R_{n}^{(2)}(F \otimes H), f_{1} \otimes f_{2}\right) \\
= & \sum_{n=-\infty}^{\infty}\left(T_{-n} F, f_{1}\right)\left(\mathscr{F} \bar{f}_{2}\right)(n)
\end{aligned}
$$

converges absolutely by Ref. 16, Theorem 5.5. By Ref. 15, Appendix 1, Theorem 3.7, the series $\Sigma_{n} T_{-n}^{(1)} R_{n}^{(2)}(F \otimes H)$ converges unconditionally in $S^{2^{\circ}}$ sense. It also follows from Ref. 15, Appendix 1, 4.14 that $T$ is a continuous linear mapping from $S^{*}$ into $S^{2^{*}}$, and we have $F=0$ if $T F=0$. Similar things hold if we consider $T$ as a mapping from $S^{\prime}$ into $\left(S^{2}\right)^{\prime}$. In the case $f \in S, T f$ can be identified with the function

$$
\sum_{n=-\infty}^{\infty} e^{-2 \pi i n w} f(z-n) \quad\left[(z, w) \in \mathbb{R}^{2}\right] .
$$

3.2. Part of the following theorem is taken from Ref. 4; for the sake of completeness we include a proof. We also note that part (i) occurs in a more abstract version in the proof of Ref.27, Chap. 1, Sec. 5, Lemma 4.

Theorem: (i) $T$ maps $L^{2}(\mathbb{R})$ isometrically onto $L^{2}\left([0,1]^{2}\right)$. (ii) Let $1 \leqslant p<2$. Then $T$ maps $L^{p}(\mathbb{R})$ into $L^{p}\left([0,1]^{2}\right)$, and the operator norm $\leqslant 1 ; T$ is injective but not surjective.

Proof: (i) Let $f \in L^{2}(\mathbb{R})$. Since the functions $f(z-n) e^{-2 \pi i n w}$ are orthogonal over $[0,1]^{2}$ we see that

$$
\begin{aligned}
\int_{0}^{1} \int_{0}^{1}|(T f)(z, w)|^{2} d z d w \\
\quad=\sum_{n=-\infty}^{\infty} \int_{0}^{1} \int_{0}^{1}\left|f(z-n) e^{-2 \pi i n w}\right|^{2} d z d w \\
\quad=\int_{-\infty}^{\infty}|f(z)|^{2} d z .
\end{aligned}
$$

Hence $T$ is well defined as a mapping of $L^{2}(\mathbf{R})$ into $L^{2}\left([0,1]^{2}\right)$, and it is norm-presa-jing.

Now let $g \in L^{2}\left([0,1]^{2}\right)$, and let

$$
c_{n m}:=\int_{0}^{1} \int_{0}^{1} g(z, w) e^{2 \pi i m z+2 \pi i n w} d z d w
$$

for integers $n$ and $m$. Putting $f(z-n):=\Sigma_{m} c_{n m} e^{-2 \pi i n z}$ for $0 \leqslant z<1$ and integer $n$, we easily see that $f \in L^{2}(\mathbb{R})$, and that $T f=g$.

(ii) Let $f \in L^{1}(\mathbb{R})$. Then

$$
\begin{aligned}
& \int_{0}^{1} \int_{0}^{1}|(T f)(z, w)| d z d w \\
& \quad \leqslant \int_{0}^{1} \int_{0}^{1} \Sigma_{n}|f(z-n)| d z d w=\int_{-\infty}^{\infty}|f(z)| d z .
\end{aligned}
$$

Hence $T f \in L^{1}\left([0,1]^{2}\right)$ and $\|T f\|_{1} \leqslant\|f\|_{1}$. It follows from convexity theory that $T$ maps $L^{p}(\mathbb{R})$ into $L^{p}\left([0,1]^{2}\right)$, and that 
$\|T f\|_{p} \leqslant\|f\|_{p}$ for $f \in L^{p}(\mathbb{R}), 1 \leqslant p \leqslant 2$.

To show injectivity, let $f \in L^{p}(\mathbb{R}), f \neq 0$. Let $f_{n} \in L^{2}(\mathbb{R})$ be such that $\left|f_{n}\right| \leqslant\left|f_{n+1}\right|$ and $f_{n} \rightarrow f, T f_{n} \rightarrow T f$ a.e. By Fatou's lemma and part (i),

$$
\begin{aligned}
& \int_{0}^{1} \int_{0}^{1}|(T f)(z, w)|^{2} d z d w \\
& \quad \geqslant \limsup _{n \rightarrow \infty} \int_{0}^{1} \int_{0}^{1}\left|\left(T f_{n}\right)(z, w)\right|^{2} d z d w=\limsup _{n \rightarrow \infty}\left\|f_{n}\right\|_{2}^{2}>0 .
\end{aligned}
$$

It is trivial that $T$ is not surjective if $1 \leqslant p<2$; otherwise we would have $T\left(L^{p}(\mathbb{R})\right) \supset T\left(L^{2}(\mathbb{R})\right)$, whence $L^{p}(\mathbb{R}) \supset L^{2}(\mathbb{R})$.

Remarks: (1) There is no way to define $T$ as a mapping of $L^{p}(\mathbb{R})$ into any $L^{r}\left([0,1]^{2}\right)$ if $p>2$ (cf. Ref. 28, Chap. XII, 2, p. 102). (2) As a mapping of $L^{p}(\mathbb{R})$ into $L^{p}\left([0,1]^{2}\right)$ with $1 \leqslant p<2, T$ is not bounded below. To see this, put $f_{c}$ $:=\Sigma_{n} c_{n} \chi_{\mid n, n+1]}$ for $c=\left(c_{n}\right)_{n} \in l^{p}$. If $T$ were bounded below there would be an $m>0$ such that $\left.\left\|T f_{c}\right\| \geqslant m\left\|f_{c}\right\|_{p}=m \Sigma_{n}\left|c_{n}\right|^{p}\right)^{1 / p}$ for $c \in l^{p}$. As $\left(T f_{c}\right)(z, w)=\Sigma_{n} c_{n} e^{-2 \pi i n \omega}$ for $c \in l^{p}$, this implies that $\left\{\Sigma_{n} c_{n} e^{-2 \pi i n \omega} \mid c \in l^{P}\right\}=L^{P}([0,1])$. Contradiction.

3.3. In the list below we have $F \in S^{*}, a \in \mathbb{C}, b \in \mathbb{C}, \alpha>0$,

(1) $T\left(T_{a} F\right)=T_{a}^{(1)}(T F)$

(2) $T\left(R_{b} F\right)=R_{b}^{(1)} T_{b}^{(2)}(T F)$,

(3) $T_{1}^{(1)}(T F)=R_{1}^{(2)}(T F)$,

(4) $T_{1}^{(2)}(T F)=T F$,

(5) $T\left(N_{\alpha} F\right)$

$$
=N_{\alpha}^{(1)}\left(\sum_{n=-\infty}^{\infty} e^{-\pi n^{2} \cosh \alpha \sinh \alpha}\left(R_{i n \sinh \alpha} T_{-n \cosh \alpha} F\right) \otimes H\right),
$$

(6) $T \mathscr{F} F=e^{-2 \pi i z \omega} U T F$,

(7) $T(P F)=P^{(1)} T F$,

(8) $T(Q F)=\left(Q^{(1)}+P^{(2)}\right) T F$.

Here $U$ is (the extension of the mapping that takes $f(z, w) \in S^{2}$ into $f(w,-z)$. All formulas except (6) follow directly from a computation. To prove (6) we first take an $f \in S$. We have by definition

$$
(T \mathscr{F} f)(z, w)=\sum_{n=-\infty}^{\infty}(\mathscr{F} f)(z-n) e^{-2 \pi i n w}
$$

Observing that $(\mathscr{F} f)(z-n) e^{-2 \pi i n w}$

$=e^{-2 \pi i z u}\left(\mathscr{F} R_{z} T_{w} f\right)(-n)$, we get by the Poisson summation formula

$$
\begin{aligned}
(T \mathscr{F} f)(z, w) & =e^{-2 \pi i z w} \sum_{n=-\infty}^{\infty} e^{2 \pi i n z} f(w-n) \\
& =e^{-2 \pi i z w}(T f)(w,-z) .
\end{aligned}
$$

For the general case take a sequence $\left(f_{n}\right)_{n}$ in $S$ that converges in $S^{*}$ sense to $F$, and use continuity of $T$ (cf. 3.1).

Remarks: (1) Formula (6) and Theorem 3.2 (i) give a quick proof of Plancherel's theorem since $U$ maps $L^{2}\left([0,1]^{2}\right)$ unitarily onto $L^{2}([0,1] \times[-1,0])$. It is of course the Poisson summation formula that does the trick here. (2) If $T f$ is sufficiently well behaved we can recover $f$ and $\mathscr{F} f$ by integration. We have

$$
\begin{aligned}
f(z) & =\int_{0}^{1}(T f)(z, w) d w, \quad(F f)(-w) \\
& =\int_{0}^{1}(T f)(z, w) e^{2 \pi i z w} d z,
\end{aligned}
$$

for $z \in \mathbb{R}, w \in \mathbb{R}$.

3.4. We calculate $T G(x, y)$ and $T \psi_{n}$ for $x \in \mathbb{R}, y \in \mathbb{R}$, $n=0,1, \cdots$. We have by the formulas of 3.3

$$
\begin{aligned}
(T G(x, y))(z, w) & =\left(T\left(e^{-\pi i x y} R_{y} T_{x} g\right)\right)(z, w) \\
& =e^{\pi i x y+2 \pi i y z}(T g)(z-x, w-y),
\end{aligned}
$$

so that we need $T g$. In general, we have by the generating function of the Hermite functions (cf. 2.2),

$$
\begin{aligned}
& c_{k}\left(T \psi_{k}\right)(z, w) \\
& \quad=C_{t^{k}}\left[e^{\pi z^{2}-2 \pi(z-t)^{2}} \sum_{n} e^{-\pi n^{2}+2 \pi i n(w+i z-2 i t)}\right] \\
& \quad=C_{t^{\wedge}}\left[\theta_{3}(w+i z-2 i t) \sum_{l=0}^{\infty} c_{l} t^{l} \psi_{l}(x)\right] .
\end{aligned}
$$

Here $\theta_{3}(z)=\Sigma_{n} \exp \left(-\pi n^{2}+2 \pi i n z\right)$ is the 3rd theta function [in the notation of Ref. 29 we have $\theta_{3}(z)=\vartheta\left(\pi z, e^{-\pi}\right)$ ]. By the Taylor expansion of $\theta_{3}$ around the point $w+i z$ we get $\left(T \psi_{k}\right)(z, w)=\sum_{l=0}^{k} \frac{\psi_{k-1}(z)}{\sqrt{ } l !}\left(\begin{array}{l}k \\ l\end{array}\right)^{1 / 2}\left(-\pi^{-1 / 2} i\right)^{l} \theta_{3}^{(l)}(w+i z)$.

In particular,

$$
(T G(x, y))(z, w)=(G(x, y))(z) \theta_{3}(w+i z-y-i x),
$$

and in case $n$ and $m$ are integers we get by 3.3,

$$
(T G(n, m))(z, w)=(-1)^{n m} e^{2 \pi i m z+2 \pi i n w} e^{-\pi z^{*}} \theta_{3}(w+i z) .
$$

As another example, let $e_{a}(t):=e^{-2 \pi i a t}$ where $a \in \mathbb{R}$. We have

$$
T\left(e_{a}\right)=\sum_{n=\infty}^{\infty} e_{a} \otimes \delta_{n-a} .
$$

Hence, if $a$ is an integer, $T\left(e_{a}\right)=e_{a} \otimes \Sigma_{n} \delta_{n}$, and if $f \in S^{*}$ is periodic with period one, then $T f=f \otimes \Sigma_{n} \delta_{n}$. Similarily, if $f$ is a function of the form $f=\Sigma_{n} c_{n} \delta_{n}$, then $T f=e^{-2 \pi i z u}$ $\left(\Sigma_{n} \delta_{n}\right) \otimes \mathscr{F} f$.

3.5. It is easy to see that $T f$ has a zero in $[0,1]^{2}$ if $T f$ is continuous and $f$ is real-valued, or even, or odd, or a Gabor function. The following theorem shows this is general.

Theorem: Let $f \in L^{2}(\mathbb{R})$ be such that $T f$ is continuous. Then $T f$ has a zero in $[0,1]^{2}$.

Proof $^{30}$ : Assuming $(T f)(z, w) \neq 0$ for $(z, w) \in[0,1]^{2}$ we can write

$$
(T f)(z, w)=e^{2 \pi i \varphi(z, w),},
$$

where $\varphi: R^{2} \rightarrow \mathbb{C}$ is continuous. Indeed, this follows at once from Ref. 31, Part VI, Sec. 1, Lemma 7.

We have by 3.3

$$
\begin{aligned}
& (T f)(z+1, w)=e^{2 \pi i w}(T f)(z, w), \\
& (T f)(z, w+1)=(T f)(z, w),
\end{aligned}
$$

for $(z, w) \in[0,1]^{2}$. Hence, for some integers $k$ and $l$

$$
\begin{aligned}
& \varphi(z+1, w)=\varphi(z, w)+w+k, \\
& \varphi(z, w+1)=\varphi(z, w)+l,
\end{aligned}
$$

for $(z, w) \in[0,1]^{2}$. Calculating $\varphi(1,1)$ in two different ways, we 
get $\varphi(0,0)+k+l=\varphi(1,1)=\varphi(0,0)+k+l+1$.

Contradiction.

Remark: If $f=\chi_{(0,1)}$, then $|(T f)(z, w)|=1$ for all $(z, w) \in \mathbb{R}^{2}$.

3.6. In the remainder of this section we determine $T(S), T\left(S^{*}\right), T(\mathrm{~S})$, and $T\left(\mathrm{~S}^{\prime}\right)$. We first give a formula which will also be used in Sec. 4 to answer questions about completeness (also cf. Ref. 6).

Theorem: Let (1) $F \in S^{*}, g \in S$ or (2) $F \in S^{\prime}, g \in S$ or (3) $F \in L^{2}(\mathbf{R}), g \in L^{2}(\mathbb{R})$. Then

$$
\sum_{n, m}\left(F, R_{-m} T_{-n} g\right) e^{2 \pi i n \omega+2 \pi i m z}=T F \cdot \overline{T g},
$$

where the identity is to be interpreted in $S^{2^{\circ}}$ for case (1) and in $\left(S^{2}\right)^{\prime}$ sense for case (2). For case (3) the identity must be interpreted in the sense that the $(m n)$ th Fourier coefficient of $T F \cdot \overline{T g}$ equals $\left(F, R_{-m} T_{-n} g\right)$.

Proof: First take $F \in S, g \in S$. Noting that

$$
\left(F, R_{-m} T_{-n} g\right) e^{2 \pi i m z}=\left(\mathscr{F} T_{z}\left(F \cdot \overline{T_{-n} g}\right)\right)_{(m)}
$$

for all $n$ and $m$ we get by the Poisson summation formula (applied to the summation over $m$ )

$$
\begin{aligned}
& \sum_{n, m}\left(F, R_{-m} T_{-n} g\right) e^{2 \pi i n w+2 \pi i m z} \\
& =\sum_{n, m} F(m+z) \overline{g(-n+m+z)} e^{2 \pi i n \omega} .
\end{aligned}
$$

Now the formula easily follows by first summing over $n$ and then over $m$.

For the general case (i.e., $\left.F \in S^{*}\right)$ take a sequence $\left(F_{k}\right)_{k}$ in $S$ which converges to $F$ in $S^{*}$ sense. It follows as in the proof of Ref. 16, Lemma 5.2 that for every $\epsilon>0$ there are positive numbers $M$ and $\beta$ such that

$$
\left|\left(F_{k}, R_{-m} T_{-n} g\right)\right| \leqslant M\left\|N_{\beta} F_{k}\right\|_{2} \exp \left(\pi \epsilon\left(n^{2}+m^{2}\right)\right),
$$

for all $n, m$, and $k$ (note that $N_{\beta} F_{k} \in S$ for $\beta>0$ ). Since $\left\|N_{\beta} F_{k}\right\|_{2}$ is bounded in $k$ for every $\beta>0$ it is not hard to complete the proof of the theorem for case (1).

The proof for case (2) is similar to that for case (1). For the proof of case (3) we take $F_{k}$ and $g_{k}$ in $S$ with $F_{k} \rightarrow F, g_{k} \rightarrow g$ in $L^{2}(\mathbb{R})$ sense. Now we note that $T F_{k} \cdot \overline{T g_{k}} \rightarrow T F \cdot \overline{T g}$ in $L^{1}\left([0,1]^{2}\right)$ and use the result already proved with $F_{k}$ and $g_{k}$ in the role of $F \in S, g \in S$. Hence, the $(m n)$ th Fourier coefficient of $T F \cdot \overline{T g}$ is given by $\left(F, R_{-m} T_{-n} g\right)$.

3.7. Theorem: $T(S)$ equals the set of all entire functions $\varphi$ of two variables such that $\varphi(z+1, w)=e^{-2 \pi i w} \varphi(z, w)$, $\varphi(z, w+1)=\varphi(z, w)$ for all $(z, w) \in \mathbb{C}^{2}$, and such that there are $M>0, A>0, B>0$ with

$$
|\varphi(x+i y, u+i v)| \leqslant M \exp \left(2 \pi x v+\pi A y^{2}+\pi B v^{2}\right) .
$$

Furthermore, $T(\mathrm{~S})$ equals the set of all $\varphi \in C^{\infty}\left(\mathrm{R}^{2}\right)$ such that $\varphi(z+1, w)=e^{-2 \pi i w} \varphi(z, w), \varphi(z, w+1)=\varphi(z, w)$ for all $(z, w) \in \mathbb{C}^{2}$. Finally,

$T\left(S^{*}\right)=\left\{F \in S^{2^{*}} \mid T_{1}^{(1)} F=R_{1}^{(2)} F, T_{1}^{(2)} F=F\right\}$, and $T\left(\mathrm{~S}^{\prime}\right)=\left\{F \in\left(\mathrm{S}^{2}\right)^{\prime} \mid T_{1}^{(1)} F=R_{1}^{(2)} F, T_{1}^{(2)} F=F\right\}$.

Proof: Let $f \in S$. It is clear that $T f$ is an entire function of two variables. Take $K>0, C>0$, and $D>0$ such that

$$
|f(x+i y)| \leqslant K \exp \left(-\pi C x^{2}+\pi D y^{2}\right) \quad(x \in \mathbb{R}, y \in \mathbb{R}) .
$$

Then for real $x, y, u, v$,

$$
\begin{aligned}
& \left|\Sigma_{n} f(x+i y-n) e^{-2 \pi i n(u+i v)}\right| \\
& \quad \leqslant K \Sigma_{n} \exp \left(-\pi C(x-n)^{2}+\pi D y^{2}+2 \pi n v\right) \\
& \quad=K \exp \left(2 \pi x v+\pi v^{2} / C+\pi D y^{2}\right) \Sigma_{n} \\
& \quad \times \exp \left(-\pi C(x+v / C-n)^{2}\right),
\end{aligned}
$$

whence $T(S)$ is contained in the set mentioned in the theorem. Conversely, let $\varphi$ be an entire function of two variables such that $\varphi(z+1, w)=e^{-2 \pi i w} \varphi(z, w), \varphi(z, w+1)=\varphi(z, w)$ for all $(z, w) \in \mathbb{C}^{2}$, and assume that $M>0, A>0, B>0$ are such that

$$
|\varphi(x+i y, u+i v)| \leqslant M \exp \left(2 \pi x v+\pi A y^{2}+\pi B v^{2}\right) .
$$

Put $\psi(z)=\int_{0}^{1} \varphi(z, w) d w$ for $z \in \mathbb{C}$. Then $\psi$ is an entire function for which $|\psi(x+i y)| \leqslant M \exp \left(\pi A y^{2}\right)$. Also,

$$
\int_{0}^{1} \varphi(x, w) d w=\int_{0}^{1} \varphi(x-[x], w) e^{-2 \pi i[x] w} d w .
$$

Let $t \in[0,1], n \in \mathbb{Z}$. We have by analyticity and periodicity of $\varphi$ in its second variable and by the estimates on $\varphi$

$$
\begin{aligned}
& \left|\int_{0}^{1} \varphi(t, w) e^{-2 \pi i n w} d w\right| \\
& =\left|\int_{0+i \gamma}^{1+i \gamma} \varphi(t, w) e^{-2 \pi i n w} d w\right| \\
& \quad \leqslant M \exp \left(2 \pi t \gamma+\pi B \gamma^{2}+2 \pi n \gamma\right)
\end{aligned}
$$

for all real $\gamma$. Minimizing with respect to $\gamma$ gives

$$
\left|\int_{0}^{1} \varphi(t, w) e^{2 \pi i n w} d w\right| \leqslant M \exp \left(-\pi B^{-1}(t+n)^{2}\right) .
$$

Hence $\psi(x)=O\left(\exp \left(-\pi B^{-1} x^{2}\right)\right)(x \in \mathbb{R})$. It follows easily from the Phragmén-Lindelöf theorem that $\varphi \in S$. It is trivial that $T \psi=\varphi$.

The proof for the $\mathrm{S}$ case is similar and will be omitted.

To prove the assertion about $\left.T S^{*}\right)$ let $F \in S^{2^{*}}$ satisfy $T_{1}^{(1)} F=R{ }_{1}^{(2)} F, T_{1}^{(2)} F=F$. For any $\psi \in S, \overline{T \psi}$ is a multiplicator of $S^{2 *}$ and it is easy to see that $F \cdot \overline{T \psi}$ is an element of $S^{2}$ which is periodic in its both variables. Hence $F . \overline{T \psi}$ has a Fourier series $\Sigma_{n, m} c_{n m}(\psi) e^{2 \pi i m z}+2 \pi i n \omega$ (cf. Ref. 3, 27.24.3). Define $G$ by $(G, \psi):=c_{00}(\psi)$. Then $G \in S^{*}$, and by Theorem 3.6, the $(n m)$ th Fourier coefficient of $T G \cdot \overline{T \psi}$ equals $\left(G, R_{-m} T_{-n} \psi\right)=c_{n m}(\psi)$. Hence $(F-T G) \cdot \overline{T \psi}=0$ for all $\psi \in S$. To show that this implies $F_{1}:=F-T G=0$, let $\psi \in S$, $\psi \neq 0$. We see from the formula

$$
T\left(R_{-b} T_{-a} \psi\right)=e^{2 \pi i b z}(T \psi)(z-a, w-b)
$$

that

$F_{1} \cdot \overline{(T \psi)(\bar{z}-a, \bar{w}-b)} \exp \left(-\pi(z-a)^{2}-\pi(w-b)^{2}\right)=0$

for all $a$ and $b$. Putting $f(z, w)=\exp \left(-\pi z^{2}-\pi w^{2}\right)$

$\left(\overline{T \psi(\bar{z}, \bar{w})}\right.$, we have $f \in S^{2}, F_{1} \cdot T_{a}^{(1)} T_{b}^{(2)} f=0$ for all $a \in \mathbb{R}, b \in \mathbb{R}$. So, if $h \in S^{2}$, then (cf. Ref. 16, Sec. 5)

$$
\begin{aligned}
0= & \left(F_{1} \cdot T_{a}^{(1)} T_{b}^{(2)} f, h\right)=\left(R_{-a}^{(1)} R_{-b}^{(2)} \mathscr{F} f, \mathscr{F}\left(h \cdot \bar{F}_{1}\right)\right) \\
= & \int_{-\infty}^{\infty} \int_{-\infty}^{\infty} e^{2 \pi i a z+2 \pi i b w} \\
& \quad \times(\mathscr{F} f)(z, w) \overline{\left(\mathscr{F}\left(h \cdot \bar{F}_{1}\right)\right)(z, w)} d z d w
\end{aligned}
$$

for all real $a$ and $b$. As $f \neq 0$, this implies that $\mathscr{F}\left(h \cdot \bar{F}_{1}\right)=0$. 
We conclude that $F_{1}=0$.

3.8. Example: Let $(a, b) \in \mathbb{R}^{2}, k=0,1, \cdots, l=0,1, \cdots$. It will be of some interest to know which $F \in S^{\prime}$ satisfies

$$
T F=\Sigma_{n, m} T_{n}^{(1)} R_{n}^{(2)} T_{m}^{(2)}\left(\delta_{a}^{(k)} \otimes \delta_{b}^{(l)}\right)
$$

[note that the right-hand side is indeed in $T\left(\mathrm{~S}^{\prime}\right)$ ]. For convenience take $(a, b) \in(0,1)^{2}$, and let $\varphi \in S$ have support in $(n, n+1)$ where $n$ is an integer. Then $(T \varphi)(z, w)=\varphi(z-n) e^{-2 \pi i n \omega}$, and $T F \cdot T \psi$ is the periodic distribution of two variables for which the restriction to $[0,1]^{2}$ equals $\overline{T_{-n} \varphi} \cdot \delta_{a}^{(k)} \otimes R_{-n} \delta_{b}^{(l)}$. The 00 th Fourier coefficient of this function is given by

$$
\begin{aligned}
\left(\delta_{a}^{(k)}, T_{-n} \varphi\right) \cdot(-2 \pi i n)^{l} e^{-2 \pi i n b} & \\
= & \left(\delta_{a-n}^{(k)}, \varphi\right)(-2 \pi i n)^{l} e^{-2 \pi i n b} .
\end{aligned}
$$

This suggests that $F=\Sigma_{n=-\infty}^{\infty}(-2 \pi i n)^{l} e^{-2 \pi i n b} \delta_{a-n}^{(k)}$, and indeed, it can be verified directly that this is the $F$ we are looking for.

\section{BARGMANN TRANSFORM, ZAK TRANSFORM, AND COMPLETENESS}

This section contains material that completes and elucidates the results of Refs. 1-7 about completeness of coherent states and expansions of the Gabor type. The Zak transform gives rise to more general results than the Bargmann transform does in the sense that with the former completeness properties for other functions than Gauss functions can be proved. On the other hand, the Zak transform is only useful when lattices of which the cells have unit area are considered.

We start this section by drawing some conclusions from Theorems 3.5 and 3.6. In particular, $L^{2}$ convergence of certain expansions of the Gabor type for well-behaved functions is proved. We consider, of course, the case in which Gabor functions are taken as basic functions in detail. The results of Refs. 2 and 7 about completeness if one or more coherent states are deleted are improved. Also, a connection with a result of J. M. Whittaker about interpolation over the lattice points in the complex plane is made. We finally indicate how the results can be extended to the cases with general lattices with cell area equal to one in the phase plane, and we show existence of expansions of the Gabor type for elements of $S^{*}$.

4.1. Theorem 3.6 is useful for analyzing the mapping $f \rightarrow\left(\left(f, R_{-m} T_{-n} g\right)\right)_{n, m}$, where $g$ is some fixed function. If, e.g., $T g$ is continuous and $1 \leqslant p \leqslant 2$, then

$\left(\left(f, R_{-m} T_{-n} g\right)\right)_{n, m} \in l^{q}$ for $f \in L^{p}(\mathbb{R})$ ( $q$ conjugate exponent), but the mapping is not bounded below (as a mapping from $L^{p}(\mathbb{R})$ into $\left.l^{q}\right)$ by Theorem 3.5.

This has an interesting signal-theoretic consequence. The function $S_{g} f$ given by $\left(S_{g} f\right)(x, y):=\left(f, R_{-y} T_{-x} g\right)$ is sometimes called a spectrogram of $f$. Hence, if we sample $S_{g} f$ over a lattice of which the cells have area one, then it may happen that the double sequence of sample values have small $q$ norm while both $f$ and $T f$ have large $p$ norm.

In case $f=g$ we have

$$
\left(f, R_{-y} T_{-x} f\right)=e^{-\pi i x y} \mathrm{Amb}(x, y ; f),
$$

where $\operatorname{Amb}(\cdot, \cdot ; f)$ is the so-called ambiguity function ${ }^{32}$ of $f$, defined by

$$
\operatorname{Amb}(x, y ; f)=\int_{-\infty}^{\infty} e^{-2 \pi i y t} f\left(t+\frac{1}{2} x\right) \overline{f\left(t-\frac{1}{2} x\right)} d t
$$

We derive from Theorems 3.5 and 3.6 the following inequality: if $f \in L^{2}(\mathbf{R}), T f$ continuous, then $\mathrm{Amb}(0,0 ; f)<\Sigma_{(n, m) \neq(0,0)}|\mathrm{Amb}(n, m ; f)|$, which is an inequality expressing the uncertainty principle. ${ }^{32,33}$

Note, however, that the assumption " $T f$ continuous" is essential, for if $f=\chi_{[0,1]}$, then $\operatorname{Amb}(0,0 ; f)=1$, $\operatorname{Amb}(n, m ; f)=0[(n, m) \neq(0,0)]$.

Taking $g=f_{-}$, where $f_{-}(t)=f(-t)$, we get

$\left(f, R_{-y} T_{-x} f_{-}\right)=\frac{1}{2} e^{-\pi i x y} W\left(\frac{1}{2} x, \frac{1}{2} y ; f, f\right)$,

where $W(\cdot, ; f f)$ is the Wigner distribution of $f$ defined by

$$
W(x, y ; f)=\int_{-\infty}^{\infty} e^{-2 \pi i y t} f\left(x+\frac{1}{2} t\right) \overline{f\left(x-\frac{1}{2} t\right)} d t .
$$

Hence the Fourier coefficients of $T f \cdot \overline{T f}$ - equal $\frac{1}{2}(-1)^{n m} \mathbf{W}\left(\frac{1}{2} n, \frac{1}{2} m ; f\right)$. As $\left(T f_{-}\right)(z, w)=(T f)(-z,-w)$, we see that it may well happen that $\mathrm{W}\left(\frac{1}{2} n, \frac{1}{2} m ; f\right)=0$ for all integers $n$ and $m$. This is seen from the Fig. This can happen even if $f \in S$, but not if $f \in S$ (cf. Theorem 3.7).

4.2. If we take a $g \in L^{2}(\mathbb{R})$ for which the set of zeros of $T g$ has measure zero, we get the completeness results obtained in Ref. 5: the set $\left(R_{-m} T_{-n} g\right)_{n, m}$ is complete in $L^{2}(\mathbb{R})$. If, in addition, $T g \in L^{q}\left([0,1]^{2}\right)$, where $q \geqslant 2$, then $\left(R_{-m} T_{-n} g\right)_{n, m}$ is complete in $L^{p}(\mathbb{R})$, where $p=q /(q-1)$. This can be proved by using Theorem 3.2 and generalizing Theorem 3.6 properly. And if $T_{g}$ is, e.g., continuously differentiable [which implies by Theorem 3.5 that $1 / \overline{T g} \notin L^{2}\left([0,1]^{2}\right)$, then $\left(R_{-m} T_{-n} g\right)_{(n, m) \neq(0,0)}$ is still complete in $L^{2}(\mathbb{R})$ [and probably also in $L^{p}(\mathbb{R})$ for $\left.1<p<2\right]$. Finally, if $f \in L^{2}(\mathbb{R}), g \in L^{2}(\mathbb{R})$ and the set of zeros of $T g$ has positive measure, then either $\left(f, R_{-m} T_{-n} g\right)=0$ for all $n$ and $m$ or $\left(f, R_{-m} T_{-n} g\right) \neq 0$ for infinitely many $n$ and $m$.

Remark: Let $g \in \mathrm{S}$. We can use Theorem 3.6 for describing the set of all $f \in \mathrm{S}^{\prime}$ such that $\left(f, R_{-m} T_{-n} g\right)=0$ in case $T g$ has a finite number of zeros $\left(a_{1}, b_{1}\right), \ldots,\left(a_{k}, b_{k}\right)$ in the unit square. By Theorem 3.6 $T f$ is concentrated in $\left(a_{1}, b_{1}\right), \ldots,\left(a_{k}, b_{k}\right)$, and the translates of these points over distances $(n, m)$ with integer $n$ and $m$. Using Ref. 34, Chap. 24, Theorem 2.4.6 (the restriction to the unit square of) we see that $T f$ has the form

$$
\sum_{i=1}^{k} \sum_{i=0}^{p_{l}} \sum_{j=0}^{q_{i}} c_{l i j} \delta_{a_{l}}^{(i)} \otimes \delta_{b_{l}}^{(j)}
$$

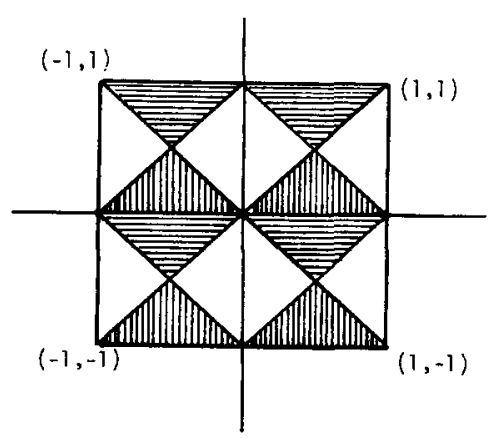

FIG. 1. If the support of $T f$ is in the \|\|$\|$ region, then the support of $T f_{-}$is in the $\equiv$ region. 
Now Example 3.8 can be applied, and we see in particular that $f$ is concentrated in the set $\left\{a_{i}+n \mid i=1, \ldots, k ; n\right.$ integer .

4.3. In Ref. 4 it was shown that for any $F \in S^{\prime}$ there exists a (not unique) expansion in a Gabor series, with convergence in $S^{\prime}$ sense. If $F \in S$, say, then it may still be the case that the convergence of the series is not in $L^{2}$ sense. The next application of Theorems 3.5 and 3.6 deals with improving convergence of series of the Gabor type. Take $f \in L^{2}(\mathbb{R}), g \in L^{2}(\mathbb{R})$ and assume that $T g \in L^{\infty}\left([0,1]^{2}\right)$. Let $(a, b) \in[0,1]^{2}$. We have for integers $n$ and $m$

$T\left(R_{-m-b} T_{-n-a} g\right)=e^{2 \pi i b z}(T g)(z-a, w-b) e^{2 \pi i m z+2 \pi i n w}$.

Hence, if $\left(c_{n m}\right)_{n, m} \in l^{2}$, then $f=\Sigma_{n, m} c_{n m} R_{-m-b} T_{-n-a} g$ (in $L^{2}(\mathbb{R})$ sense) if and only if

$$
(T f)(z, w)=e^{2 \pi i b z}(T g)(z-a, w-b) \sum_{n, m} c_{n m} e^{2 \pi i m z+2 \pi i n w}
$$

[in $L^{2}\left([0,1]^{2}\right)$ sense]. Now, if $T f$ and $T g$ are continuous, then it is in general advisable to take $a$ and $b$ such that

$(T f)(z, w)=0$ whenever $(T g)(z-a, w-b)=0$. By Theorem 3.5 this is always possible if $T g$ has only one zero in $[0,1]^{2}$. If $a$ and $b$ are such that $T f / T\left(R_{-b} T_{-a} g\right) \in L^{2}\left([0,1]^{2}\right)$, then one can take for $c_{n m}$ the $(m, n)$ th Fourier coefficient of $T f / T\left(R_{-b} T_{-a} g\right)$. We see in particular (cf. 3.4) that a considerable class of functions $f$ have an expansion $f=\Sigma_{n, m} c_{n m} G(n+a, m+b)$ in an $L^{2}(\mathbb{R})$ convergent Gabor series for suitable values of $a$ and $b$ (this class contains all functions $f$ for which $T f$ is Hölder continuous).

Note: We can use the Zak transform to prove existence of Gabor type expansions for tempered distributions in general. Let $g \in \mathrm{S}, g \neq 0$ be such that $T g$ is real analytic and has no zeros on the edges of the unit squares (the latter assumption is probably superfluous, but convenient). According to Lojasiewicz's theorem ${ }^{35}$ there exists a distribution $\Phi$ of two variables such that $\Phi \cdot T g=T f$. This $\Phi$ is, in general, not periodic, but by our assumption on $g$ we may assume it is. Hence $\Phi$ has a Fourier series expansion $\Sigma_{n, m} c_{n m} e^{2 \pi i n z}+2 \pi i m w$, and it follows that $f=\Sigma_{n m} c_{n m} R_{-n} T_{-m} g$. This generalizes Ref. 4, Theorem 4.7 where the Gabor case was considered [however, in the proof of the theorem quoted a method is given to determine the double sequence $\left.\left(c_{n m}\right)_{n, m}\right]$.

4.4. We now turn to the Gabor case in detail. We know from Ref. 7, 2.14 that any regular tempered distribution $f$ with $(f, G(n, m))=0$ for all integers $n$ and $m,(n, m) \neq(0,0)$ is a multiple of the function $f_{0}$ given by

$$
f_{0}(t)=2^{-1 / 4} \exp \left(\pi t^{2}\right) \sum_{n-1}(-1)^{n} \exp \left(-\pi\left(n-\frac{1}{2}\right)^{2}\right)
$$

This function which can also be found in Ref. 6, is an interesting one as we shall see. We let $g(t)=2^{1 / 4} \exp \left(-\pi t^{2}\right)$.

Theorem: For every $p, 1 \leqslant p<\infty$ we have

$f_{0} \in L^{\infty}(\mathbb{R}) \backslash L^{p}(\mathbb{R})$. The Bargmann transforms $\left(B f_{0}\right)(z)$ of $f_{0}$ equals $-\theta_{1}(z) e^{i \pi z^{2}} / 2 \pi z$, where $\theta_{1}$ is the first theta function [in the notation of Ref. 29 we have $\left.\theta_{1}(z)=\vartheta_{1}\left(\pi z, e^{-\pi}\right)\right]$. We further have $\mathscr{F} f_{0}=f_{0}$, and $T f_{0}=d / \overline{T g}$ where $d=-\frac{1}{2} \vartheta ;\left(0, e^{-\pi}\right)$.

Proof: It was already observed in the proof of Ref. 7, Theorem 2.14 that $f_{0}$ is bounded. Now let $1 \leqslant p<\infty$. We have for $t \geqslant 0$

$$
\left|f_{0}(t)\right|=2^{-1 / 4} \exp \left(\pi t^{2}-\pi \varphi^{2}(t)\right)+0(\exp (-2 \pi t)),
$$

where $\varphi(t)=\left[t+\frac{1}{2}\right]-\frac{1}{2}$. If $n=1,2, \cdots$, then

$$
\begin{aligned}
\int_{n}^{n+!} & \exp \left(\pi p t^{2}-\pi p \varphi^{2}(t)\right) d t \\
& =\int_{n-!}^{n+!} \exp \left(\pi p t^{2}-\pi p\left(n+\frac{1}{2}\right)^{2}\right) d t \\
& \geqslant \exp (-p \pi)\left[\left(n+\frac{1}{2}\right)-\left(\left(n+\frac{1}{2}\right)^{2}-1\right)^{1 / 2}\right] \\
& \geqslant \exp (-p \pi) /(2 n+1) .
\end{aligned}
$$

It follows that $f_{0} \notin L^{p}(\mathbb{R})$. that

To show that $\left(B f_{0}\right)(z)=-\theta_{1}(z) e^{\left\lfloor\pi z^{2}\right.} / 2 \pi z$, we observe

$$
P\left(g \cdot f_{0}\right)=-\sum_{n=-\infty}^{\infty}(-1)^{n} e^{-\pi(n \cdots \cdots !)^{n}} \delta_{n-!} / 2 \pi i
$$

Taking Fourier transforms and using that $\mathscr{F} P=Q \mathscr{F}$, we get $^{36}$

$$
-2 \pi i z\left(\mathscr{F}\left(g \cdot f_{0}\right)\right)(z)=\sum_{n=-\infty}^{\infty}(-1)^{n} e^{-\pi(n-1)-2 \pi i n-1) z} .
$$

The right-hand side is equal to $i \vartheta_{1}\left(\pi z, e^{-\pi}\right)$. Also

$$
\left(\widetilde{F}\left(g \cdot f_{0}\right)\right)(z)=\left(R_{z} f_{0}, g\right)=e^{-\pi z^{2}}\left(T_{-i z} f_{0}, g\right) .
$$

Now using Jacobi's identity $\vartheta_{1}\left(\pi z, e^{-\pi}\right)$

$$
\begin{gathered}
=i \exp \left(-\pi z^{2}\right) \vartheta_{1}\left(\pi i z, e^{-\pi}\right)(\text { Ref. } 29,21.51) \text { we get } \\
-2 \pi i z e^{-\pi z^{2}}\left(T_{-i z} f_{0}, g\right)=e^{-\pi z^{2}} \vartheta_{1}\left(\pi i z, e^{-\pi}\right) .
\end{gathered}
$$

It follows from the oddness of $\vartheta_{1}$ that

$$
\left(T_{z} f_{0}, g\right)=-\vartheta_{1}\left(\pi z, e^{-\pi}\right) / 2 \pi z
$$

Now use the definition of $B$.

We next show that $T f_{0}=d / \overline{T g}$. As it stands, this relation must be considered in distributional sense since $f_{0} \notin L^{p}(\mathbb{R})$ for $1 \leqslant p \leqslant 2$. We have $\left(f_{0}, G(n, m)\right)=0$ for all integers $n$ and $m$ with $(n, m) \neq(0,0)$, and according to what we have found above,

$$
-2 \pi z\left(R_{z} f_{0}, g\right)=\vartheta_{1}\left(\pi z, e^{-\pi}\right),
$$

so that (cf. Ref. 29, 21.41)

$$
\begin{aligned}
\left(f_{0}, g\right) & =\left.\frac{-1}{2 \pi} \frac{d}{d z}\left[\vartheta_{1}\left(\pi z, e^{-\pi}\right)\right]\right|_{z=0} \\
& =-\frac{1}{2} \vartheta_{1}\left(0, e^{-\pi}\right) \\
& =-\frac{1}{2} \vartheta_{2}\left(0, e^{-\pi}\right) \vartheta_{3}\left(0, e^{-\pi}\right) \vartheta_{4}\left(0, e^{-\pi}\right) \neq 0 .
\end{aligned}
$$

Applying Theorem 3.6 we get

$$
T f_{0} \cdot \overline{T g}=-\frac{1}{2} \vartheta_{1}^{\prime}\left(0, e^{-\pi}\right)=d .
$$

Since $T f_{0} \in\left(S^{2}\right)^{\prime}$ and $T g$ has a zero in $\left(\frac{1}{2}, \frac{1}{2}\right)$ and nowhere else in $[0,1]^{2}$, there is a $\varphi \in\left(S^{\prime}\right)$ concentrated in the points $\left(n+\frac{1}{2}\right.$, $\left.m+\frac{1}{2}\right)$ with integers $n$ and $m$ such that $T f_{0}=d / \overline{T g}+\varphi[$ by Theorem 3.7 we see that $d / \overline{T g}] \in T\left(\mathrm{~S}^{\prime}\right)$. It follows from the remark in Sec. 4.2 that $\varphi=T f_{1}$, where $f_{1}$ is concentrated in the points $n+\frac{1}{2}$, with $n$ an integer. However,

$d / \overline{T g_{1}}=\overline{T k}$, where $k$ is the regular distribution given by

$$
k(z)=\int_{0}^{1} \frac{d d w}{\left(T g_{1}\right)(z, w)} .
$$

Here we use that if $\psi: \mathbb{R}^{2} \rightarrow \mathbb{C}$ is absolutely integrable over 
$[0,1]^{2}$ and satisfies $T_{1}^{(1)} \psi=R_{1}^{(2)} \psi, T_{1}^{(2)} \psi=\psi$, then $\psi=T \psi_{0}$, where $\psi_{0}(z)=\int_{0}^{l} \psi(z, w) d w$ (this follows from Theorem 3.6). Now the fact that $f_{0}$ and $k$ are regular and $f_{1}$ is concentrated in the points $n+\frac{1}{2}$ with integer $n$ leads to a contradiction, unless $\varphi \equiv 0$. This shows that $T f_{0}=d / \overline{T g}$.

We finally show that $\mathscr{F} f_{0}=f_{0}$. We therefore observe that $T f_{0}=d / \overline{T g}=d / \overline{T \mathscr{F}^{*}} g=d e^{2 \pi i z w} /(\overline{T g)(w,-z})$ by 3.3,(6). Hence $\left(T \mathscr{F} f_{0}\right)(z, w)=e^{-2 \pi i z w}\left(T f_{0}\right)(z, w)$

$=d /(\overline{T g})(z, w)=\left(T f_{0}\right)(z, w)$, and the result follows from 3.1

Remarks: (1) It is easy to see that $\Sigma_{n} f_{0}(z-n) e^{--2 \pi i n w}$ converges uniformly and absolutely (to $1 / \overline{\mathrm{Tg}}$ on any compact set not containing points $\{z, w\}$ for which $z-\frac{1}{2}$ is an integer. In case $z=0$ we thus find the Fourier series for $1 / \theta_{3}$ :

$$
\left(\sum_{n}^{\infty} e^{-\pi n^{2}+2 \pi i m \omega}\right)^{\cdots 1}=d^{-1} \sum_{n=-\infty}^{\infty} f_{0}(n) e^{2 \pi i n \omega}
$$

(compare Ref. 29, Chap. 21, Miscellaneous Examples, 14). Note also that $d^{-1} f_{0}(r-s)$ for integers $r$ and $s$ is the limit of the $(r s)$ th element of the inverse of the matrix $\left(e^{-m k-l^{2}}\right)_{k, l=-n, \cdots, n}$ if $n \rightarrow \infty$; these matrices occur in the study of the inverses of discrete Gauss transforms. ${ }^{37}$ (2) As a consequence of Theorem 4.4 we have that the set $(G(n, m))_{(n, m) \neq(0,0)}$ is complete in $L^{p}(\mathbb{R})$ if $1 \leqslant p<\infty$, and not complete in $L^{\infty}(\mathbb{R})$. This generalizes the result in Ref. 2 where the case $p=2$ was treated. (3) Let $f \in L^{1}(\mathbb{R})$ so that for every integer $m$

$$
c_{n m}:=(-1)^{n m} d^{-1}\left(f, R_{-m} T_{-n} f_{0}\right) \rightarrow 0
$$

if $n \rightarrow \infty$. Since for all integers $k$ and $l$

$d^{-1} \Sigma_{n, m}(-1)^{n m}(G(n, m), G(k, l)) R_{-m} T_{-n} f_{0}=\overline{G(k, l)}$ where the series converges boundedly, we have

$$
\sum_{n . m} c_{n m}(G(n, m), G(k, l))=(f, G(k, l)) .
$$

It follows from Ref. 4, Theorem 4.1 and 4.1, Remark 1 that $f=\Sigma_{n, m} c_{n, m} G(n, m)$ with convergence in $S^{\prime}$ sense.

4.5. In Ref. 2 it is shown that the set $G(n, m)$ with $(n, m) \neq(0,0),(n, m) \neq\left(k_{0}, l_{0}\right)$ is not complete in $L^{2}(\mathbb{R})$ if $\left(k_{0}, l_{0}\right) \neq(0,0)$. We generalize this result fo $L^{p}(\mathbb{R})$ as follows. If $k$ and $l$ are integers and $F \in \mathrm{S}^{\prime}$, then $R_{-k}^{(1)} R_{-1}^{(2)}, T F$

$=T\left(R,{ }_{k} T_{. l} F\right)$ by 3.3. So, if $P(z, w)=\Sigma_{k, l} c_{l k} e^{2 \pi i k z+2 \pi i l w}$ is a trigonometric polynomial, then

$$
T\left(\Sigma_{k, l} c_{l k} R_{-k} T_{-l} f_{0}\right)=P / \overline{T g} .
$$

Taking $P$ such that $P\left(\frac{1}{2}, \frac{1}{2}\right)=0$, we get $P / \overline{T_{g} \in L}{ }^{\infty}\left([0,1]^{2}\right)$. Since $T$ maps $L^{2}(\mathbb{R})$ onto $L^{2}\left([0,1]^{2}\right) \supset L^{\infty}\left([0,1]^{2}\right)$, we see that there is an $f \in L^{2}(\mathbb{R})$ such that $T f=P / \overline{T g}$, i.e., an $f \in L^{2}(\mathbb{R})$ with $(f, G(k, l))=(-1)^{k l} c_{k l}$. Taking $c_{00}=1$,

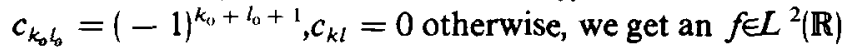
with $(f, G(n, m))=0$ for $(n, m) \neq(0,0),(n, m) \neq\left(k_{0}, l_{0}\right)$. This $f$ is given by

$$
f=f_{0}+(-1)^{k_{11}+l_{11}+1} R_{-k_{0}} T_{-\zeta} f_{0},
$$

and it can be shown that $f \in L^{p}(\mathbb{R})$ for all $p$ with $1 \leqslant p \leqslant \infty$. Hence, the collection $G(n, m)$ with $(n, m) \neq\left(k_{0}, l_{0}\right)$ is not complete in $L^{p}(\mathbb{R})$ for $1 \leqslant p \leqslant \infty$. In case $l_{0}=0$ we can even show that $f(x)=\left((-1)^{k_{11}+1} e^{2 \pi i k_{11} x}+1\right) f_{0}(x)=O(1 /|x|)(|x| \rightarrow \infty)$ and that $f$ is continuous. The discontinuities of $f_{0}$ (which occur at the points $x$ for which $x-\frac{1}{2}$ is an integer) are annihilated by the factor $(-1)^{k_{0}+1} e^{2 \pi i k_{10} x}+1$ in this case. And if $k_{0}=0$ we get an $f$ for which $\left(\mathcal{F}^{\prime} f\right)(x)=O(1 /|x|)(|x| \rightarrow \infty)$ and $\mathcal{F} f$ is continuous. One can carry this process further, and it is easy to see that deletion of more coherent states gives rise to the existence of smoother functions perpendicular to all coherent states but the deleted ones. We note that the latter assertion is, to some extent, also true if one takes more general functions $g$ than the Gauss function.

4.6. We now discuss an expansion which is in some sense dual to Gabor's expansion. Let $A$ be a finite set of lattice points, and let $F \in \mathrm{S}^{\prime}$ satisfy $(F, G(n, m))=0$ for $(n, m) \notin A$. It is clear that $F$ is of the form

$$
F=G+d^{-1} \sum_{(n, m) \in A}(-1)^{n ! m}(F, G(n, m)) R{ }_{m} T_{-, n} f_{0},
$$

where $(G, G(n, m))=0$ for all $n$ and $m$ [this is a consequence of the formula $\left.\left(R_{-m} T_{-n} f_{0}, G(k, l)\right)=(-1)^{n m} d \delta_{n k} \delta_{m l}.\right]$ In case $F \in S^{\prime}$ is regular and satisfies $\Sigma_{n, m}|(F, G(n, m))|<\infty$, then we can take for $A$ all of $\mathbb{Z} \times \mathbb{Z}$ and $G \equiv 0$ :

$$
\left(^{*}\right) F=d \Sigma_{n, m}(-1)^{n m}(F, G(n, m)) R_{-m} T_{-n} f_{0} .
$$

For the proof we use that either function in $\left(^{*}\right)$ is regular (cf Theorem 4.4) and Ref. 4 , Theorem 4.1. Of course, $\left({ }^{*}\right)$ is false in general if $F \in S^{\prime}$ is not regular.

We shall now give a connection with Whittaker's result $^{38}$ about interpolation over the Gaussian integers. Therefore, let $F \in S^{\prime}$ be regular and assume that $\Sigma_{n, m}|(F, G(n, m))|<\infty$ so that the expansion in (*) holds for $F$. Applying the Bargmann transform to both sides and using that

$$
\begin{aligned}
& \left(B f_{0}\right)(z)=-\theta_{1}(z) e^{\frac{1}{2} \pi z^{2}} / 2 \pi z, \\
& B\left(R_{-m} T_{-n} f_{0}\right)(z)=(-1)^{n m} e^{-1 \pi\left(n^{2}+m^{2}\right)+\pi(n+i m \mid z} \\
& \times\left(B f_{0}\right)(z+i m-n) \text {, } \\
& \theta_{1}(z+i m-n) e^{\left.\frac{1}{2 \pi} \pi+i m-n\right)^{2}} \\
& =(-1)^{n+m+n m} \theta_{1}(z) e^{\left\lfloor\pi z^{2}\right.} e^{-\left\{m\left(n^{2}+m^{2}\right)-\pi(n+i m) z\right.}, \\
& (F, G(n, m))=e^{-\frac{1}{2} \pi\left(n^{2}+m^{2}\right)}(B F)(n-i m),
\end{aligned}
$$

we get

$$
B\left(R_{-m} T_{-n} f_{0}\right)(z)=\frac{(-1)^{n+m+1} \theta_{1}(z) e^{\frac{l}{2} \pi z^{2}}}{2 \pi(z+i m-n)},
$$

so that

$$
\begin{aligned}
(B F)(z)= & -d \theta_{1}(z) e^{\frac{1}{2} \pi z^{2}} \sum_{n, m}(-1)^{n+m+n m} \\
& \times \frac{(B F)(n-i m)}{z-n+i m} e^{-\frac{1}{2}\left(n n^{2}+m^{2}\right)} .
\end{aligned}
$$

This is a slight generalization of the result of Ref. 38, because there only functions $f=B F$ are admitted that have growth $<(2, \pi / 2)$, while we admit certain functions with growth $=(2, \pi / 2)$ (cf. 2.7).

The expansion discussed here can be used to generalize a result of Iyer and Pfluger ${ }^{39}$ about entire functions of growth $<(2, \pi / 2)$ which are bounded at the lattice points. Let $\varphi=B f$, where $\mathrm{f} \in \mathrm{S}$, and assume that $\varphi$ is bounded at the lattice points. We shall show that $\varphi$ is constant, and to that 
end we suppose that $\varphi$ has type $\pi / 2$. Since $z^{k} \varphi(z)=\left(B(Q-i P)^{k} f\right)(z) \in B(\mathrm{~S})$, we see that

$$
\begin{aligned}
z^{k} \varphi(z)= & -d \theta_{1}(z) e^{i m z^{2}} \sum_{n, m}(-1)^{n+m+n m} \\
& \times \frac{(n+i m)^{k} \varphi(n+i m)}{z-n-i m} e^{-\frac{1}{2} m\left(n^{2}+m^{2}\right)}
\end{aligned}
$$

for all $k=0,1, \cdots$. Since $\varphi \in B(\mathrm{~S})$ we know that $\sum_{n=0}^{\infty}\left|a_{n}\right||z|^{n}=O\left(\exp \left(\frac{1}{2} \pi|z|^{2}\right)(1+|z|)^{-K}\right)$ for all $K>0$ [here we write $\varphi(z)=\sum_{n=0}^{\infty} a_{n} z^{n}$ ]. It follows easily that

$$
\begin{aligned}
& \varphi^{2}(z)=\sum_{k=0}^{\infty} a_{k} z^{k} \varphi(z) \\
& =-d \theta_{1}(z) e^{i \pi z^{2}} \Sigma_{n, m}(-1)^{n+m+n m} \frac{\varphi^{2}(n+i m)}{z-n-i m} \\
& X e^{-\frac{1}{2} m n^{2}+m^{2} \mid} .
\end{aligned}
$$

Now the right-hand side has growth $\leqslant(2, \pi / 2)$, while the lefthand side has growth $=(2, \pi){ }^{40} \mathrm{We}$ conclude that $\varphi$ has type $<\pi / 2$, whence $\varphi$ is constant by the result of Iyer and Pfluger.

A trivial extension of this theorem is: If $\varphi \in B(\mathrm{~S})$ satisfies $\varphi(n+i m)=O\left((n+i m)^{K}\right)$ for some $K=0,1, \cdots$, then $\varphi$ is a polynomial of degree $\leqslant K$. As a consequence we have: If $f \in \mathrm{S}$ and $(f, G(n, m))=O\left(\left(n^{2}+m^{2}\right)^{K / 2} \exp \left(-\frac{1}{2} \pi\left(n^{2}+m^{2}\right)\right)\right.$, then $f$ is of the form $\Sigma_{k=0}^{K} a_{k} \psi_{k}$. We observe (cf. 4.5) that the assumption " $f \in S$ " cannot be weakened to a condition in which boundedness of only finitely many of the functions $Q^{k} P^{l} f$ is required.

With methods similar to the ones used above it can be shown that if $f \in \mathrm{S}, 0 \leqslant \epsilon_{0}<1$, $(f, G(n, m))=O\left[\exp \left(-\frac{1}{2} \pi \epsilon\left(n^{2}+m^{2}\right)\right)\right]$ for all $\epsilon, 0<\epsilon<\epsilon_{0}$, then $f \in N_{\alpha}\left(S^{*}\right)$, where $\alpha=-\frac{1}{2} \log \left(1-\epsilon_{0}\right)$.

4.7. Translating Ref. 4 , Theorem 4.7 by using the Bargmann transform, we get the following theorem:

Theorem: Let $f \in B\left(S^{\prime}\right)$, i.e., let $f$ be an entire function such that

$$
f(z) \exp \left(-\frac{1}{2} \pi|z|^{2}\right)=O\left((1+|z|)^{N}\right)
$$

for some $N>0$. There exists a double sequence $\left(c_{n m}\right)_{n m}$ satisfying $c_{n m}=O\left(\left(1+n^{2}+m^{2}\right)^{K}\right)$ for some $K>0$ such that

$$
f(z)=\Sigma_{n, m} c_{n m} \exp \left(-\frac{1}{2} \pi\left(n^{2}+m^{2}\right)\right) e^{\pi(n+i m) z} .
$$

The convergence of the series is such that

$$
\begin{aligned}
& \mid f(z)-\sum_{n^{2}+m^{2}<L} c_{n m} \exp \left(-\frac{1}{2} \pi\left(n^{2}+m^{2}\right)\right) \\
& \times e^{m(n+i m \mid z} \mid \frac{\exp \left(-\frac{1}{2} \pi|z|^{2}\right)}{(1+|z|)^{M}} \rightarrow 0
\end{aligned}
$$

uniformly in $z \in \mathbb{C}(L \rightarrow \infty)$ for some $M>0$. Write $f(z)=e^{1 \pi|z|^{2}} \varphi(z)$. If $\varphi \in L^{2}(\mathbb{C})$ and

$$
\int_{\sigma}\left|\frac{\varphi(z)}{z-w}\right| d z \rightarrow 0 \quad(|w| \rightarrow \infty),
$$

then there exists a unique sequence $\left(c_{n m}\right)_{n m}$ with $c_{n m} \rightarrow 0\left(n^{2}+m^{2} \rightarrow \infty\right)$ such that the above expansion holds; these $c_{n m}$ 's are given by

$c_{n m}$

$$
=-(-1)^{n+m+n m} d^{-1} \int_{G} e^{\left.l i m|z|^{2}-\bar{z}^{2}\right)} \frac{\theta_{1}(\bar{z}) \varphi(z)}{2 \pi(\bar{z}-n-i m)} d z .
$$

Proof: Take $h \in \mathrm{S}^{\prime}$ such that $f=B h$. This $h$ has a Gabor expansion $h=\Sigma_{n, m} c_{n m} G(n, m)$ which converges in $S^{\prime}$ sense. Now apply $B$ to both sides and use 2.7 and 2.8 . The convergence is in the sense indicated by Ref. 13, Sec. 4.1.

Assume in addition that $\varphi \in L^{2}(\mathbb{C})$ and that

$$
\int_{\mathbf{C}}\left|\frac{\varphi(z)}{z-w}\right| d z \rightarrow 0 \quad(|w| \rightarrow \infty)
$$

where $\varphi$ is as in the theorem. Put $f_{\alpha}=B N_{\alpha} h$ for $\alpha>0$ (cf. 2.4). As $N_{\alpha} h \in S$ for $\alpha>0$ we have $N_{\alpha} h=\Sigma_{n, m} c_{n m}(\alpha) G(n, m)$, where

$$
c_{n m}(\alpha)=(-1)^{n m} d^{-1}\left(N_{\alpha} h, R_{-n} T_{-m} f_{0}\right)
$$

by 4.4 , Remark 3 . By 4.6 and 2.8 we can write this as

$c_{n m}(\alpha)$

$$
=-(-1)^{n+m+n m} d^{-1} \int_{\mathrm{C}} e^{-\pi|z|^{2}} f_{\alpha}(z) \overline{\frac{\theta_{1}(z) e^{\pi z^{2} / 2}}{2 \pi(z+i m-n)}} d z .
$$

Since $\left(B N_{\alpha} h\right)(z)=\exp \left(-\frac{1}{2} \alpha+\frac{1}{2} \pi\left|z e^{-\alpha}\right|^{2}\right) \varphi\left(z e^{-\alpha}\right)$ by $2.8(3)$ and $\theta_{1}(z)=O\left(\exp \left(\pi(\operatorname{Im} z)^{2}\right)\right)$, we get

$$
\begin{aligned}
& \lim _{\alpha \downarrow 0} c_{n m}(\alpha) \\
& =-(-1)^{n+m+n m} d^{-1} \int_{C} e^{\underline{z} \pi\left(\vec{z}^{2}-|z|^{2} \mid\right.} \frac{\theta_{1}(\bar{z}) \varphi(z)}{2 \pi(\bar{z}-n-i m)} d z
\end{aligned}
$$

boundedly in $(n, m)$ as $\int_{\mathbb{C}}|\varphi(z)| /|z-w| d z$ is bounded in $w$. We also have $c_{n m} \rightarrow 0$ and

$$
f(z)=\Sigma_{n, m} c_{n m} \exp \left(-\frac{\pi}{2}\left(n^{2}+m^{2}\right)\right) e^{\pi(n+i m) z} .
$$

Uniqueness follows from Ref. 4, Theorem 4.1, for it follows from the assumptions about $\varphi$ and 2.7 that $h \in L^{2}(\mathbb{R})$.

4.8. We give an explicit formula for the unique coefficients $c_{n m}(x, y)$ in the Gabor expansion $\Sigma_{n, m} c_{n m}(x, y) G(n, m)$ of $G(x, y)$, with $c_{n m}(x, y) \rightarrow 0\left(n^{2}+m^{2} \rightarrow \infty\right)$. Using the formulas

$$
\begin{aligned}
& c_{n m}(x, y)=-(-1)^{n m} d^{-1}\left(G(x, y), R_{-m} T_{-n} f_{0}\right), \\
& \left(T_{a} R_{b} G(x, y), f_{0}\right)=\exp (\pi i(a y-x b))\left(G(x-a, y-b), f_{0}\right), \\
& \left(G(x-a, y-b), f_{0}\right) \\
& =\exp \left(-\frac{1}{2} \pi(x-a)^{2}-\frac{1}{2} \pi(y-b)^{2}\right) \overline{\left(B f_{0}\right)(x-a-i(y-b)),} \\
& \left(B f_{0}\right)(z)=-d \theta_{1}(z) e^{\frac{l}{l \pi z^{2}} / 2 \pi z,} \overline{\theta_{1}(z)}=\theta_{1}(\bar{z}),
\end{aligned}
$$

we get

$$
\begin{aligned}
& \left(G(x, y), R_{-b} T_{-a} f_{0}\right) \\
& =-d \exp \left(-\pi(y-b)^{2}-\pi i(x y+a b)\right) \\
& \quad \times \theta_{1}(x-a+i(y-b)) / 2 \pi(x-a+i(y-b)) .
\end{aligned}
$$

Hence,

$$
\begin{aligned}
c_{n m}(x, y)= & \exp \left(-\pi(y-m)^{2}-\pi i x y\right) \\
& \times \theta_{1}(x-n+i(y-m)) / 2 \pi(x-n+i(y-m))
\end{aligned}
$$

for all real $x$ and $y$ and all integers $n$ and $m$.

If we denote for $f \in S$ by $c_{n m}(f)$ the unique Gabor coefficients in the expansion $f=\Sigma_{n, m} c_{n m}(f) G(n, m)$, then we see from Ref. 3, 27.12.1.5 that

$$
c_{n m}(f)=\iint(f, G(x, y)) c_{n m}(x, y) d x d y
$$


In particular,

$$
\iint(G(a, b), G(x, y)) c_{n m+1}(x, y) d x d y=c_{n m}(a, b)
$$

which shows that $c_{n m}$ is an "eigenfunction" of the kernel operator with kernel $(G(a, b), G(x, y))$.

4.9. We next consider the completeness problem with general lattices in phase plane where the cells have area equal to one. Such a lattice can be described by six real numbers $a_{11}, a_{12}, a_{13}, a_{21}, a_{22}, a_{23}$, with $a_{11} a_{22}-a_{12} a_{21}=1$, by putting

$\left(x_{n, m}, y_{n, m}\right)=\left(a_{11} n+a_{12} m+a_{13}, a_{21} n+a_{22} m+a_{23}\right)$

for integers $n$ and $m$. We have according to Ref. 3, 27.12.2.1, for all lattice points $\{a, b\}=\left(x_{n, m}, y_{n, m}\right\}$

$$
\begin{aligned}
& \left(\Gamma_{\left(A, \psi^{\prime}\right)} f, R_{-b} T_{-a}\left(\Gamma_{\mid A, \psi\}} g\right\}_{-}\right) \\
& =\frac{1}{2} e^{-\pi i a b} W\left(\frac{1}{2} a, \frac{1}{2} b ; \Gamma_{(A, \psi\}} f, \Gamma_{(A, \psi)} g\right) \\
& =\frac{1}{2} e^{-\pi i a b} W\left(\frac{1}{2} n, \frac{1}{2} m ; f, g\right)=(-1)^{n m} e^{-\pi i a b}\left(f, R_{-m} T_{-n} g\right)
\end{aligned}
$$

for $f \in S, g \in S$ (cf. 4.1 for the definition of $W$ ). Here $\Gamma_{(A, \psi)}$ is the special transform introduced in Ref. 3,27.3.8 and 9 associated with the matrix

$$
\left[\begin{array}{ccc}
a_{22} & -i a_{21} & -i a_{23} / 2 \\
i a_{21} & a_{11} & a_{13} / 2 \\
0 & 0 & 1
\end{array}\right]
$$

The above formula can easily be generalized to the case that $f \in S^{*}, g \in S$ or $f \in S^{\prime}, g \in S$ or $f \in L^{2}(\mathbb{R}), g \in L^{2}(\mathbb{R})$. Hence, characterizing the set of all $f \in S^{\prime}$ for which $\left(f, R_{-b} T_{-a} g\right)=0$ for all lattice points $(a, b)=\left(x_{n, m}, y_{n, m}\right)$ amounts to characterizing all $F \in S^{\prime}$ for which $\left(F, R_{-m} T_{-n} G_{-}\right)=0$ for all integers $n$ and $m$, where $G=\Gamma_{(\vec{A}, \dot{1})}^{-1} g$.

As an example take $g(t)=2^{1 / 4} \exp \left(-\pi t^{2}\right)$ and $a_{13}=a_{23}=0$. We have

$$
\left(\Gamma_{\{A, \psi\}}^{-1} g\right)(z)=2^{1 / 4} a_{22}{ }^{-1 / 2} \exp \left(-\pi z^{2}\left(1+i a_{21} a_{22}\right) / a_{22}^{2}\right)
$$

if $a_{12}=0$, and

$$
\begin{aligned}
& \left(\Gamma_{\left.\mid \bar{A} \cdot k_{1}\right)}^{-1} g\right)(z) \\
= & 2^{1 / 4}\left\{a_{22}-i a_{12}\right)^{-1 / 2} \exp \left(-\pi z^{2}\left[\frac{a_{12}}{a_{22}^{2}\left(a_{12}+i a_{22}\right)}+\frac{i a_{11}}{a_{12}}\right]\right)
\end{aligned}
$$

if $a_{12} \neq 0$ (the choice of the square root is determined by $\psi$; cf. Ref. 3, 27.3). Now if $\operatorname{Re} \gamma>0, g_{\gamma}(t)=\exp \left(-\pi \gamma t^{2}\right)$, then a calculation shows that

$$
\left(T g_{\gamma}\right)(z, w)=\exp \left(-\pi \gamma z^{2}\right) \vartheta_{3}\left(\pi(w+i \gamma z) ; e^{-\pi \eta}\right) .
$$

It follows that $T g_{\gamma}$ has zeros at the points $\left(n+\frac{1}{2}, m+\frac{1}{2}\right)$ with integers $n$ and $m$ and nowhere else. Now the remark in Sec. 4.2 gives an indication of the general form of all $F \in S^{\prime}$ with $\left(F, R_{-m} T_{-n} g_{\gamma}\right)=0$ for all $n$ and $m$. In this case, however, we can do better by using the Bargmann transform. Let $\gamma \neq 0$. We want to characterize all $F \in S^{\prime}$ 'with $(F, G(n+\gamma m, m))=0$ for all integers $n$ and $m$. That is, we must find all $F \in S^{\prime}$ such that $(B F)(n+\gamma m-i m)=0$ for all integers $n$ and $m$. Note that the function $\vartheta_{1}\left(\pi z, e^{-\pi i \gamma-\pi}\right)$ has zeros at the points $n+\gamma m-i m$, that there is an $M>0$ such that

$$
\left|\vartheta_{1}\left(\pi z, e^{-\pi i \gamma-\pi}\right)\right| \leqslant M \exp \left(\pi(\operatorname{Im} z)^{2}\right) \quad(z \in \mathbb{C}),
$$

and that there is a $K>0$ such that

$$
\left|\vartheta_{1}\left(\pi z, e^{-\pi i \gamma-\pi}\right)\right| \geqslant K \exp \left(\pi v^{2}\right)
$$

where $z=-\gamma v+i v+a-\frac{1}{2}$ with $v$ real and $a$ an even integer (compare Ref. 7, 2.6). Now we can proceed as in the proof of Ref. 7, 2.11 to conclude that $(B F)(z)$ $=e^{i \pi z^{2}} \vartheta_{1}\left(\pi z, e^{-\pi i \gamma-\pi}\right) P(z)$, where $P$ is a polynomial. The only difference from the proof quoted is that we need a theorem of Phragmén-Lindelöf type for regions of the form $(\varphi=\arctan \gamma)$ :

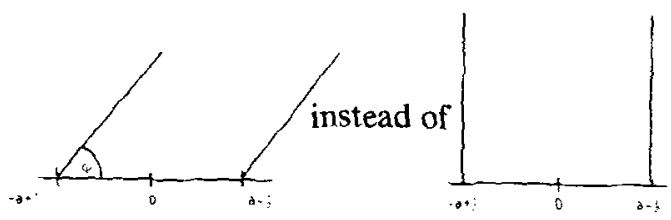

With the aid of the Schwarz-Christoffel formula one can construct a conformal mapping $\tau$ (continuous at the boundaries) that maps the second region onto the first one such that $\tau\left(-a+\frac{1}{2}\right)=-a+\frac{1}{2}, \tau\left(a-\frac{1}{2}\right)=a-\frac{1}{2}$, $\tau(i \infty)=(-\gamma+i) \cdot \infty$, and such that $|\tau(x+i w) / w|$ tends to a finite limit $\neq 0$ uniformly in $x,|x| \leqslant a-\frac{1}{2}$ if $w \rightarrow \infty$. Hence, the Phragmén-Lindelof theorem for the rectangular region can be modified in such a way that we get the required bounds on $e^{-\frac{1}{2} \pi z^{2}}(B F)(z) / \vartheta_{1}\left(\pi z, e^{-\pi i \gamma-\pi}\right)$. From this we can derive a similar characterization as in Ref. $7,2.12$. We note that the more general problem of characterizing all $F \in S^{\prime}$ with $\left(F, G\left(n \alpha+\gamma m, m \alpha^{-1}\right)\right)=0$ for all integers $n$ and $m$ can be handled similarly $(\alpha>0)$. Finally, if we have a general lattice $(a, b)=\alpha n\left(\cos \theta_{1}, \sin \theta_{1}\right)+\beta m\left(\cos \theta_{2}, \sin \theta_{2}\right)$ with $\alpha \beta \sin \left(\theta_{2}-\theta_{1}\right)=1$, and $(F, G(a, b))=0$, then we can use that

$$
(B F)(z)=c\left(B N_{e} \cdot{ }_{i a} F\right)\left(e^{i \theta_{1}} z\right) \quad(z \in \mathbb{C})
$$

for some $c,|c|=1$ (cf. 2.8 and the references given there). We then get $(F, G(a, b))=0$ at the lattice points if and only if $\left(N_{e} \quad{ }_{i \theta} F, G\left(n \alpha+\beta m \cos \left(\theta_{2}-\theta_{1}\right), m \alpha^{-1}\right)\right)=0$ for all integers $n$ and $m$.

4.10. We finally show that every $F \in S^{*}$ can be expanded in an $S^{*}$ convergent Gabor series. Our proof consists of a suitable variation of the argument used in the proof of Ref. 4 , Theorem 4.7, where the $S^{\prime}$-case was considered.

We start with the observation that any $F \in S$ " which can be written in the form

$$
\left(^{*}\right) F=\sum_{n=0}^{\infty} a_{n}(Q+i P)^{n} F_{n},
$$

where $F_{n} \in L^{2}(\mathbb{R}),\left\|F_{n}\right\| \leqslant 1, a_{n}=O\left(\exp \left(-\frac{1}{2} n \log n-B n\right)\right)$ for all $B>0$ has a Gabor representation. Indeed, as $F_{n} \in L^{2}(\mathbb{R})$ we can find $\left(c_{k i}^{(n)}\right)_{k, l}$ such that

$$
F_{n}=\Sigma_{k, l} c_{k l}^{(m)} G\{k, l\}
$$

(convergence in $S^{\prime}$ sense). Here we may assume in addition that

$$
\left|c_{k l}^{(n)}\right| \leqslant C\left((\log |k|)^{1 / 2}+(\log |l|)^{1 / 2}\right)
$$


for some $C>0$ independent of $n$ (cf. Ref. 4, Theorem 4.6). Hence, since $(Q+i P) G(x, y)=(x+i y) G(x, y)$,

$$
F=\sum_{n=0}^{\infty} a_{n}\left(\Sigma_{k, l} c_{k l}^{(n)}(k+i l)^{n} G(k, l)\right)
$$

Now we note that

$$
\sum_{n=0}^{\infty}\left|a_{n} c_{k l}^{(n)}(k+i l)^{n}\right| \leqslant C \sum_{n=0}^{\infty} a_{n}|k+i l|^{n+1}=f(|k+i l|),
$$

where $f(z):=\Sigma_{n} a_{n} z^{n+1}$. It follows from the assumptions on $\left(a_{n}\right)_{n}$ and Ref. 21, Theorem 2.2.10 that $f$ has growth $\leqslant(2,0)$. Hence

$$
\sum_{n=0}^{\infty}\left|a_{n} c_{k l}^{(n)}(k+i l)^{n}\right|=O\left(\exp \left(\epsilon\left(k^{2}+l^{2}\right)\right)\right)
$$

for all $\epsilon>0$. We conclude that

$$
F=\Sigma_{k, l}\left(\sum_{n=0}^{\infty} a_{n} c_{k l}^{(n)}(k+i l)^{n}\right) G(k, l),
$$

with convergence in $S^{*}$ sense [this follows from the fact that for every $f \in S$ there is an $\epsilon>0$ such that $(f, G(k, l))$ $\left.=O\left(\exp \left(-\epsilon k^{2}+l^{2}\right)\right)\right]$.

Our next aim is to show that any $F \in S^{*}$ can be written in the form $\left(^{*}\right)$ with $F_{n} \in L^{2}(\mathbf{R}),\left\|F_{n}\right\| \leqslant 1, a_{n}$ $=O\left(\exp \left(-\frac{1}{2} n \log n-B n\right)\right)$ for all $B>0$. So, let $F=\Sigma_{k=0}^{\infty} c_{k} \psi_{k} \in S^{*}$, where $c_{k}=O\left(e^{k \epsilon}\right)$ for all $\epsilon>0$. Let $k_{0}$, $k_{1}, k_{2}, \cdots$ be a sequence of integers with $0=k_{0} \leqslant k_{1} \leqslant k_{2} \leqslant \cdots$, and let

$$
e_{k, l}=\pi^{1} c_{k}((k+l) ! / k !)^{-1 / 2}
$$

for $k=k_{l}, k_{l}+1, \cdots, k_{l+1}-1, l=0,1, \cdots$. The definition of the $e_{k, l}$ 's is such that

$$
\sum_{k=k_{l}}^{k_{t}+1} c_{k} \psi_{k}=(Q+i P)^{l_{t+1}} \sum_{k=k_{l}}^{-1} e_{k, l} \psi_{k+l}
$$

We have to choose the $k_{l}$ 's such that for all $B>0$,

$$
\begin{aligned}
& \| \sum_{k=k_{l}}^{k_{t+}} e_{k, l}^{-1} \psi_{k+l}||_{2} \\
& \quad=\left(\sum_{k=k_{l}}^{k_{l+1}-1}\left|e_{k, l}\right|^{2}\right)^{1 / 2}=O\left(\exp \left(-\frac{1}{2} l \log l-B l\right)\right) .
\end{aligned}
$$

Equivalently, we want the $k_{l}$ 's such that for all $B>0$,

$$
A_{l}:=\sum_{k=k_{l}}^{k_{l}+1-1} \frac{k !\left|c_{k}\right|^{2}}{(k+l) !}=O(\exp (-l \log l-B l)) .
$$

Denote for $l=0,1, \cdots$ by $f(l)$ the integer with the property that $\left|c_{k}\right| \leqslant e^{l}(k=0,1, \cdots, f(l)-1),\left|c_{f(l)}\right|>e^{l}$ [we may assume that $f(l)$ exists, otherwise $\left(c_{k}\right)_{k}$ is bounded and so $\left.F \in \mathrm{S}^{\prime}\right]$. Now $f(l) / l \rightarrow \infty$, for otherwise we could find an $M>0$ and integers $l_{1}, l_{2}, \cdots$ with $l_{k} \rightarrow \infty$ such that $f\left(l_{k}\right) \leqslant M l_{k}$, $\left|c_{f\left(l_{k}\right)}\right|>\exp \left(l_{k}\right)$ for all $k$, contradicting $c_{l}=O(\exp (l \epsilon))$ if $\epsilon<1 / M$. Now put $k_{l+1}:=\min \left(l^{2}, f,(l)\right)$ for $l=0,1,2, \cdots$ We have

$$
A_{l} \leqslant e^{2 l} \sum_{k=k_{l}}^{k_{1+}-1} \frac{k !}{(k+l) !},
$$

and it follows easily from Stirling's formula that there is an $M>0$ such that

$$
k ! /(k+l) !<M \exp \left(l-l \log l-l \log \left(1+k_{l} / l\right)\right),
$$

for $l=0,1, \cdots, k=k_{l}, \ldots, k_{l+1}-1$. Hence,

$$
A_{l} \leqslant M e^{3 l}\left(k_{l+1}-k_{l}\right) \exp \left(-l \log l-l \log \left(1+k_{l} / l\right)\right) .
$$

Since $k_{l} / l \rightarrow \infty, k_{l+1}-k_{l}=O\left(l^{2}\right)$, it follows that $A_{l}=O(\exp (-l \log l-B l))$ for all $B>0$, and this completes the proof.

We mention a consequence: It follows as in 4.7 that for every entire $f$ of growth $\leqslant(2, \pi / 2)$ there exists a double sequence $\left(c_{n m}\right)_{n m}$ with $c_{n m}=O\left[\exp \left(\epsilon\left(n^{2}+m^{2}\right)\right)\right]$ for all $\epsilon>0$ such that

$$
f(z)=\Sigma_{n, m} c_{n m} \exp \left(-\frac{\pi}{2}\left(n^{2}+m^{2}\right)\right) e^{\pi(n+i m) z} .
$$

'V. Bargmann, P. Butera, L. Girardello, J. R. Klauder, Rep. Math. Phys. 2, 221 (1971).

${ }^{2}$ A. M. Perelomov, Theor. Math. Phys. 6, 156 (1971)

${ }^{3}$ N. G. de Bruijn, Nieuw Arch. Wisk. 21, 205 (1973).

${ }^{4}$ A. J. E. M. Janssen, "Gabor representation of generalized functions," (to appear in J. Math. Anal. Appl.).

${ }^{5}$ H. Bacry, A. Grossman, and J. Zak, Phys. Rev. B 12, 1118 (1975).

"M. J. Bastiaans, "Gabor's expansion of a signal into Gaussian elementary signals," (to appear).

${ }^{7}$ A. J. E. M. Janssen, "Weighted Wigner distributions vanishing on lattices," (to appear in J. Math. Anal. Appl.).

"J. von Neumann, "Mathematical Foundations of Quantum Mechanics" (Princeton U. P., Princeton, N.J., 1955), Chap. 5, Sec. 4.

${ }^{9}$ See Refs. 1 and 2; also the case that $f \in L^{2}(\mathbb{R}), \alpha \beta<1$ was considered in both references.

${ }^{10}$ D. Gabor, J. Inst. Elec. Eng. 93, 429 (1946).

"After completing the manuscript the author was informed by J. Zak that the proof of this property has been announced before in J. Zak, Phys. Rev. B 12, 3023 (1975), and that a proof(essentially the same as the one given by the author) will appear in J. Math. Phys. See also Ref. 5 for an important special case. Zak also pointed out that in Ref. 2 and in M. Boon and J. Zak, Phys. Rev. B 18, 6744 (1978), expansions of the Gabor type were considered.

${ }^{12}$ V. Bargmann, Comm. Pure Appl. Math. 14, 187 (1961).

${ }^{13}$ V. Bargmann, Comm. Pure Appl. Math. 20, 1 (1967).

${ }^{14}$ I. M. Gel'fand, G. E. Shilov, Generalized Functions, Vol. 2 (Academic, New York, 1968), Chap. IV, Sec. 2.3.

${ }^{15}$ A. J. E. M. Janssen, "A pplications of the Wigner distribution to harmonic analysis of generalized stochastic processes," MC-tract 114, Appendix 1 (Amsterdam, 1979).

${ }^{16}$ A. J. E. M. Janssen, Proc. K. Ned. Akad. Wet. A 82, 283 (1979)

${ }^{17}$ See Ref. 3, 27.6.

${ }^{18} \mathrm{M}$. Reed and B. Simon, Methods of modern mathematical physics, Vol. 1 (Academic, New York, 1972), Appendix to Chap. V, Sec. 3. The Hermite coefficients of a tempered distribution have at most polynomial growth.

${ }^{19}$ See Ref. 3, Sec. 27.3 for generalizations.

${ }^{20}$ See Ref. 15 , Sec. 4.

${ }^{2}$ R. P. Boas, Entire Functions (Academic, New York, 1954)

${ }^{22}$ A. J. E. M. Janssen,. "Bargmann transform, carriers of generalized func tions, and the Wigner distribution," (to appear). See also Ref. 13, Sec. 6.

${ }^{23}$ J. Zak, Phys. Rev. Lett. 19, 1385 (1967).

${ }^{24} \mathrm{~J}$. Zak, Phys. Rev. 168, 686 (1968).

${ }^{25}$ J. Zak, Solid State Phys. 27, 1 (1972)

${ }^{26}$ J. Zak, Phys. Rev. 187, 1803 (1969).

${ }^{27}$ J. Igusa, Theta Functions (Springer, New York, 1972).

${ }^{2 \times}$ A. Zygmund, Trigonometric Series, Vol. II (Cambridge U. P., 1968).

${ }^{29}$ E. T. Whittaker, G. N. Watson, A Course of Modern Analysis, 4th ed. (Cambridge U. P., Cambridge, 1927).

${ }^{30}$ The author thanks Ralph Howard for proving this theorem; see also Ref. 11.

${ }^{31}$ T. Raeb and P. V. Reichelderfer, Continuous Transformations in Analysis (Springer, New York, 1955).

${ }^{32}$ R. Price and E. M. Hofstetter, IEEE Trans. Inf. Theory 11, 207 (1965).

${ }^{33}$ N. G. de Bruijn, in Inequalities, edited by O. Shisha (Academic, New 
${ }^{33}$ N. G. de Bruijn, in Inequalities, edited by O. Shisha (Academic, New York, 1967), p. 57.

${ }^{34} \mathrm{~F}$. Trèves, Topological vector spaces, distributions and kernels (Academic, New York, 1972).

${ }^{35}$ S. Lojasiewicz, Studia Math. 18, 87 (1959).

${ }^{36}$ This can be justified by using the theorems in Ref. 16, Sec. 5 .

${ }^{37}$ I. J. Good, Applicable Anal. 9, 205 (1979).

${ }^{38}$ J. M. Whittaker, Interpolatory Function Theory (Cambridge, U.P., Cam- bridge, 1935), Chap. V, Sec. 12, $\mathrm{L}_{501}$.

${ }^{39}$ V. G. Iyer, J. London Math. Soc. 13, 91 (1938); A. Pfluger, Proc. London Math. Soc. 42, 305 (1939). Both authors prove (by different methods) that any entire function of growth $<(2, \pi / 2)$ which is bounded at the lattice points $n+i m$ must be a constant. The result we prove is more general in the sense that certain functions of growth $=(2, \pi / 2)$ are allowed.

${ }^{40} \mathrm{The}$ proof given here consists of a suitable adaptation of Iyer's proof of the result stated in Ref. 39. 